\title{
Das jungpleistozäne Lößprofil von Nußloch (SW-Wand) im Aufschluss der Heidelberger Zement AG
}

\author{
Erhard Bibus, Manfred Frechen, Michael Kösel \& Wolfgang Rähle *)
}

\begin{abstract}
Kurzfassung: Im Aufschluss Nußloch südlich von Heidelberg ist ein neu aufgeschlossener Lößrücken paläopedologisch aufgenommen und ein Standardprofil mit verschiedenen Methoden untersucht worden. Im Einzelnen wurden Sedimentanalysen (Korngrößen, Kohlenstoff, Kalkgehalt) und die magnetische Suszeptibilität bestimmt, biomorphologische und malakologische Untersuchungen durchgeführt sowie 27 Proben mit der IRSL-Methode datiert. Stratigraphisch umfasst der untersuchte Abschnitt das Jungpleistozän mit einem mehrgliedrigen Eem-Boden und einem ungewöhnlich mächtigen Würmlöß (ca. $18 \mathrm{~m}$ ).

Bodentypologisch wurden ein Bt-Horizont, eine Humuszone und zahlreiche Nassböden von unterschiedlicher Intensität mit und ohne Verbraunungszone nachgewiesen. Von den fünf ausgewiesenen Verbraunungszonen (WB2 - WB6) sind WB2, WB4 und WB5 aufgrund der Molluskenführung als interstadiale Bildungen anzusehen. Hinzu kommt noch eine der drei interstadialen Mosbacher Humuszonen (WB1) im stark reduzierten Altwürmabschnitt mit einem Alter von ca. 75 ka BP. Als kräftigster Interstadialboden ist der BvHorizont einer arktischen Braunerde erkannt worden (WB4), welcher aufgrund verschiedener Kriterien mit dem Lohner Boden bei ca. 30 ka BP parallelisiert wurde. Der im Hangenden des Lohner Bodens überlieferte Jungwürmlöß ist mit $12 \mathrm{~m}$ für Mitteleuropa sehr mächtig und von zahlreichen Nassböden (mindestens 10) untergliedert. Besonders aufgespaltet ist der Bereich des E2-Nassbodens, in dem auch die Böden WB5 und WB6 mit schwachen Verbraunungszonen liegen. Auch der jüngste Jungwürmlöß über dem Leithorizont des Eltviller Tuffs ist mit 4,5 m relativ mächtig und mehrgliedrig überliefert, so dass von einem E4/E5-Komplex gesprochen wird.

Die paläopedologischen Befunde stimmen hervorragend mit den Veränderungen in der Molluskenfauna und der Suszeptibilitätskurve überein, so dass detaillierte stratigraphische und paläoklimatische Rekonstruktionen möglich sind. Die absoluten Altersdatierungen bestätigen dabei weitgehend die vorgenommenen Alterseinstufungen.

Abschließend wird das neu bearbeitete Profil in Nußloch mit Ergebnissen von ANTOINE et al. (2001) verglichen, welche im gleichen Aufschluss an anderer Stelle erzielt worden sind.
\end{abstract}

\section{[The Late Pleistocene loess profile Nussloch (SW wall)]}

Abstract: At the Nussloch section located south of Heidelberg, a more recently exposed new loess wall was pedologically investigated. The profiles were investigated multidisciplinarily including grain-size spectra, organic and carbonate content, magnetic susceptibility, as well as biomorphology and malacology and infrared optically stimulated luminescence (IRSL). The lower part of the Late Pleistocene includes the subdivided Eemian paleosol and the Wuermian loess about $18 \mathrm{~m}$ thick. A Bt horizon, a humic-rich horizon ("Humuszone") and several tundra gleysols ("Nassböden") of different intensity, with and without Bw horizons could be detected. Five brown soils (WB2 - WB6) are intercalated in the loess. The brown soils WB2, WB4 and

* Anschriften der Verfasser: Prof. Dr. E. Bibus, Geographisches Institut der Universität, Rümelinstraße 1923, D-72070 Tübingen; Prof. Dr. M. Frechen, Institut für Geowissenschaftliche Gemeinschaftsaufgaben (GGA-Institut), Stilleweg 2, D-30655 Hannover; Dr. M. Kösel, RP Freiburg, Landesamt für Geologie, Rohstoffe und Bergbau Baden-Württemberg, Dienstgeb. Stuttgart, Urbanstraße 53, D-70182 Stuttgart; Dr. W. RÄHLE, Staatliches Museum für Naturkunde, Rosenstein 1, D-70191 Stuttgart. 
WB5 correlate to interstadial periods, as evidenced by the mollusc spectra. Furthermore, WB1 correlates to one of the three Early Wuermian interstadials ("Mosbacher Humuszone"). Soil WB1 with an age estimate of about $75 \mathrm{ka} \mathrm{BP}$ represents the reduced Early Wuermian sequence.

The most intensive brown soil (WB4) has the typical soil criteria of an arctic brown soil, which most likely correlates to the about 30 ka old Lohne Soil ("Lohner Boden"). The Late Wuermian loess covering the Lohne Soil is about $12 \mathrm{~m}$ thick and intercalated by at least ten tundra gleysols. The equivalent of the E2-Nassboden forms a tundra gley complex including also the weak brown soils WB5 and WB6. The youngest Upper Wuermian loess about $4.50 \mathrm{~m}$ thick covers the Eltville tephra and is designated to correlate to the E4/E5 complex.

The paleopedological results are in excellent agreement with the results and interpretation by means of malacology and magnetic susceptibility enabling detailed stratigraphic and paleoclimatic reconstruction.

Keywords: loess, paleosol, Late Pleistocene, absolute dating, loess snails, SW Germany, magnetic susceptibility

\section{Einleitung}

Im terrestrischen Bereich wird die letzte Kaltzeit besonders gut durch mächtige Lößablagerungen mit darin eingeschalteten Bodenbildungen überliefert. In Mitteleuropa sind mit dem Profil Schwalbenberg bei Sinzig am unteren Mittelrhein (Bibus 1980, 1995; SCHIRMER 1990, 2000), den Profilen Schillo \& Co. am Gräselberg (Semmel 1968: 15), in Schierstein bei Wiesbaden (SABEL \& THEws 1986) sowie in Nußloch bei Heidelberg (Bente 1987; Bente \& LÖSCHER 1987; ZÖLLER 1995; ZÖLLER \& LÖSCHER 1999; ANTOINE et al. 2001) Lokalitäten bekannt geworden, in denen der Würmlöß zwischen 15 und $20 \mathrm{~m}$ Mächtigkeit erreicht. In Nußloch ist zudem der Altwürmlöß nur rudimentär ausgebildet, so dass das knapp 20 $\mathrm{m}$ mächtige Profil vorwiegend den Mittel- und Jungwürmlöß repräsentiert. Da solche hochauflösenden Lößabfolgen wichtige Informationen zur Paläopedologie, Paläoklimaentwicklung, Paläoökologie und Stratigraphie liefern können, wurden von uns in den Deckschichten des Muschelkalksteinbruchs der Heidelberger Zement AG in Nußloch neue Untersuchungen durchgeführt. Es handelt sich dabei nicht um die bisher beschriebenen Profile (BENTE \& LÖSCHER 1987; ZÖLLER 1995; ZÖLLER \& LÖSCHER 1999; Antoine et al. 2001; Rousseau et al. 2002; Hatté et al. 1999, 2001), sondern um den seit 1998 neu aufgeschlossenen SW-Bereich des Aufschlusses, in dem ein von Dellen flankierter Lößriedel durch den Abraumabbau vollkommen durchschnitten wurde (vgl. Abb. 1). Der Riedel verläuft ungefähr in SE-NWRichtung und setzt an einem ostexponierten Hang in ca. $205 \mathrm{~m}$ ü.NN an. Nach W erfolgt noch ein ca. $20 \mathrm{~m}$ hoher Anstieg zu einem flachen Wasserscheidenbereich in ca. 220 bis 225 $m$ ü.NN, welcher NNW-SSE verläuft. ANTOINE et al. (2001: 212) haben die Fortsetzung dieses breiten Lößrückens als eine von NNW-Winden sedimentierte Lößdüne angesehen. Der von uns bearbeitete Riedel liegt somit als Kleinform rechtwinklig zu der als Lößdüne angesehenen Form vor. Von Bedeutung ist, dass die im Löß enthaltenen Horizonte die Riedeloberfläche nachzeichnen. Der Riedel ist deshalb nicht durch Eintiefung der flankierenden Dellen, sondern durch stetige Aufschüttung entstanden. Da der Aufschluss nur ca. $3 \mathrm{~km}$ von der östlichen Bruchstufe des Rheintalgrabens entfernt liegt, ist wegen der Nähe des Auswehungsgebietes mit einer stark sandigen Lößfazies zu rechnen. Der zentrale Bereich des aufgeschlossenen Riedels wurde durch zehn eingemessene Profile in seiner Gesamtheit aufgenommen (vgl. Abb. 2). An der Stelle mit der vollständigsten Gliederung ist ein Standardprofil erstellt und für verschiedene Analysen beprobt worden (vgl. Abb. 3 bis 6). Im Einzelnen erfolgten Sedimentanalysen (Korngröße, Kalkgehalt, organische Substanz) (BIBus \& KöSEL), Schneckenbestimmungen (RÄHLE), gesteinsmagnetische Messungen (Suszeptibilität, Paläomagnetik) (APPEL), biomorphologische Analysen (GoLJEWA) und Lumineszenz-Datierungen (IRSL) (FRECHEN). 


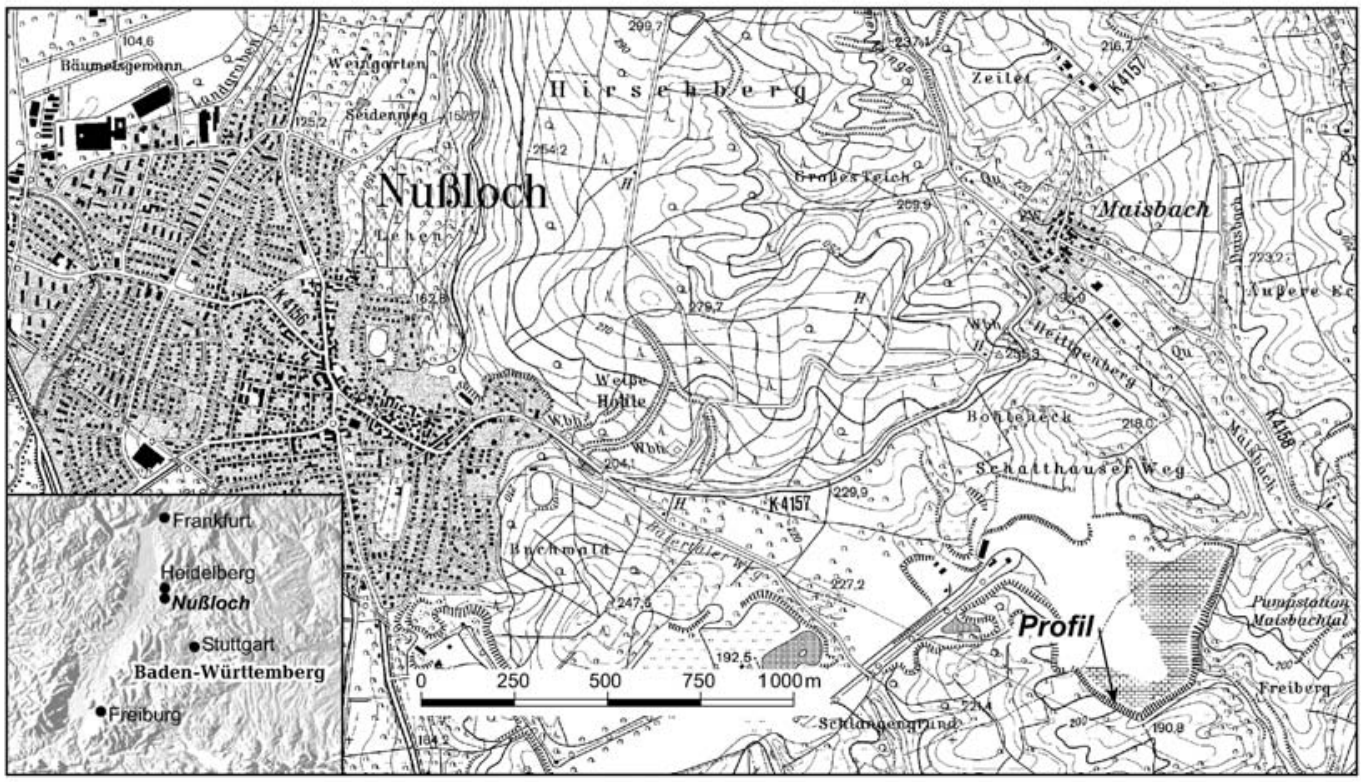

Abb. 1: Übersichtskarte mit Lage des bearbeiteten Lößriedels im Aufschluss Nußloch.

(Druck mit Genehmigung des Landesvermessungsamts Baden-Württemberg, Az. 5.12/1581)

Fig. 1: Map showing the position of the profile at the Nussloch section.

\section{Beschreibung und Interpretation des Lößriedels im zentralen Bereich}

Das aufgenommene Profil beginnt an der Basis mit einem präeemzeitlichen sandigen Kies aus Lokalschottern. Darüber folgt ein $30 \mathrm{~cm}$ mächtiger, vernässter Lößlehm mit Rost- und Bleichbändern, der nach seiner Position in die Rißeiszeit einzustufen ist. Eine Überprägung durch eine Nassbodenbildung (Bruchköbeler Böden) i.S.v. BiBus (1974) ist nicht auszuschließen. Abgeschlossen wird der rißzeitliche Lößlehm durch einen bis $1,70 \mathrm{~m}$ mächtigen, stellenweise mehrgliedrigen Bt-Horizont (Abb. 2, Abschnitt A, Schichten 46, 45, 44). In seinem oberen Bereich besteht der fossile Boden aus einem rötlichbraunen mittel schluffigen Ton mit vielen Sesquioxidkonkretionen und groben Polyedern, auf denen kräftige Tonüberzüge ausgebildet sind. Im mittleren Abschnitt (45) löst sich der Bt-Horizont in einen Lamellenstreifen-Horizont mit Bbt-Bändern auf. Dieser Zone folgt nach unten ein brauner lehmiger Schluff mit schwach polyedrischem Gefüge. In diesem Bvt-Horizont (46) sind ebenfalls noch schwach ausgebildete Lamellenstreifen zu erkennen. Der gesamte B-Horizont wird dem Eem (MIS 5e) zugeordnet. Neben sehr vielen einheitlich ausgebildeten Bt-Horizonten sind entsprechende mehrgliedrige Unterböden der letztinterglazialen Parabraunerde vereinzelt auch an anderen Stellen in Baden-Württemberg beobachtet worden (z. B. Nußloch an anderer Stelle, ZölLer \& LöScher 1999; Reihen, Bibus 2002; Bönnigheim, BiBus 2002; Gundelfingen, Bibus \& Strahl 2000: 221). Die Mehrgliedrigkeit wird von uns dadurch erklärt, dass der Eemboden einen vorstrukturierten Rißlöß z. B. mit Nassböden oder Bodensediment-Einlagerungen überprägt hat. ZÖLLER \& LÖSCHER (1999: 41) diskutieren auch die Möglichkeit, dass der untere Teil des Bodenkomplexes dem vorletztinterglazialen Boden entspricht. Das Vorkommen der Mehrgliedrigkeit nur in gewissen Reliefpositionen, das Auskeilen des mittleren Horizontes innerhalb eines Aufschlusses sowie die Unterlagerung durch typisch gegliederten Rißlöß mit den Bruchköbeler Nassbö- 


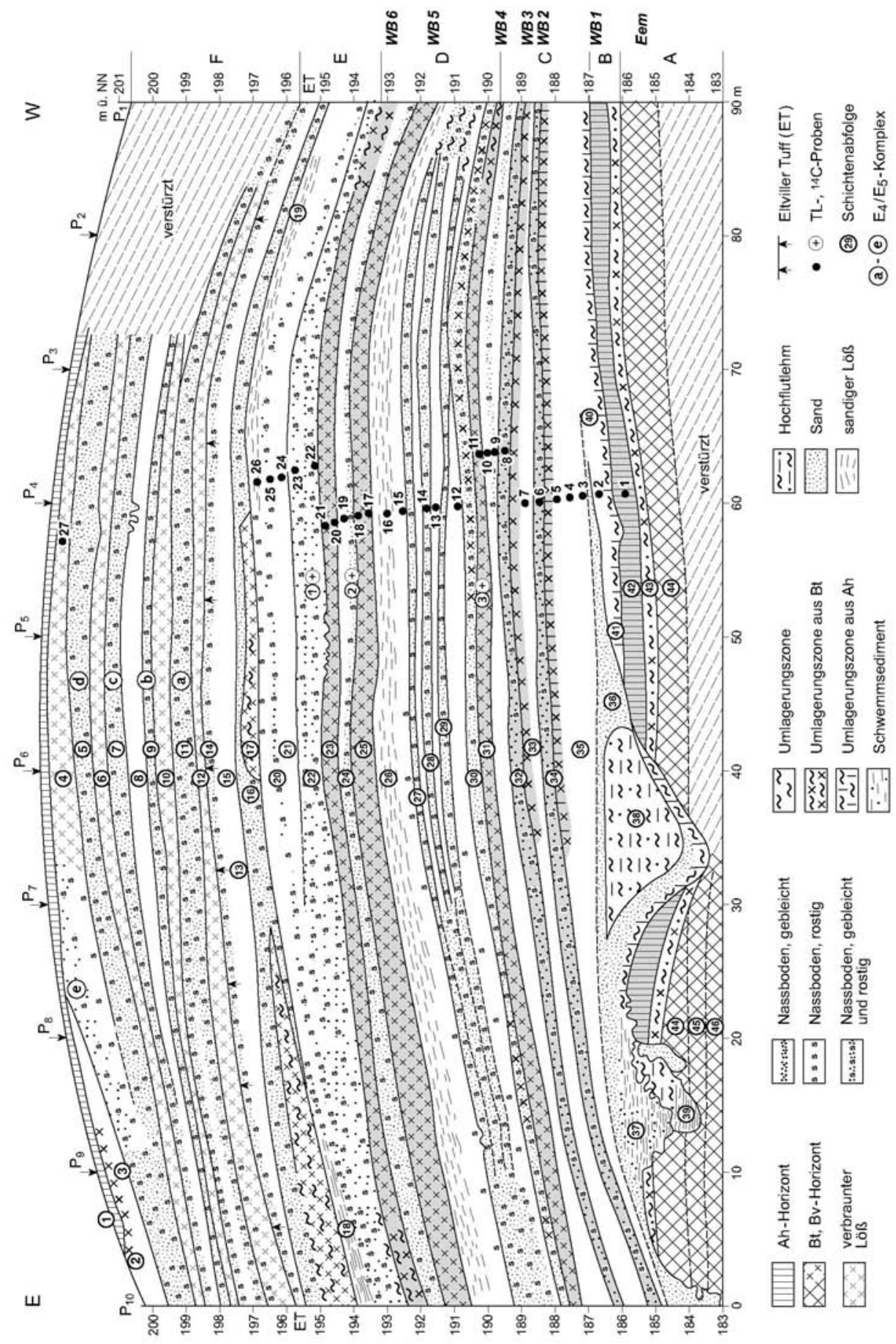

Abb. 2: Aufschlussprofil des zentralen Lößriedels im Aufschluss Nußloch.

Fig. 2: Sketch showing the profiles under study in the central part of the loess „Riedel“ at the Nussloch section. 
Erläuterung zur Abb. 2

\section{Ap-Horizont}

2 Btv-Horizont, rez. Parabraunerde

3 Jüngster Jungwürmlöß

4 Schwach verbraunter Löß, z.T. mit schwachen Vernässungserscheinungen

5 Nassboden

6 Sehr schwacher Bv-Horizont

7 Nassboden

8 Jungwürmlöß

9 Nassboden

10 Sehr schwacher Bv-Horizont

11 Nassboden (E4)

$12 \mathrm{Sehr}$ schwacher Bv-Horizont (E4)

13 Eltviller Tuff (ET)

14 Schwacher Nassboden, E3

15 Jungwürmlöß zwischen E3 und E2

16 Nassboden, z.T. mit umgelagerter Bt-Material (E2)

17 Umgelagertes Bt-Material (Kolluvium)

18 Feingebänderter Schwemmlöß

19 Jungwürmlöß, oben sandstreifig

20 Schwacher Nassboden

21 Jungwürmlöß, z.T. schwach vernässt

22 Nassboden, z.T. zweigeteilt

23 Bv-Horizont, Boden WB6

24 Vernässungszone (Nassboden)

25 Bv-Horizont, Boden WB5

26 Löß, oben sandstreifig

27 Nassboden

28 Nassboden, kräftig

29 Nassboden, verlagert, mit liegendem Löß

30 Vernässungszone (Nassboden)

31 Kräftiger Bv-Horizont mit liegendem Löß, Boden WB4, Lohner Boden

32 Nassboden mit schwachen Bv-Horizont, WB3, Gräselberger Boden 1

33 Mittelwürmlöß

34 Kräftiger Nassboden mit humosen Bv, WB2, Gräselberger Boden 2

35 Mittelwürmlöß

36 Sand

37 Sand- u. Schluffbänder, wechsellagernd, Kiese

38 Hochflutlehm

39 Schluff- u. Lehmbänder, wechsellagernd

40 Löß, schwach verbraunt

41 Fließerde aus Ah-Material

42 Mosbacher Humuszone, WB1

43 Zweigeteilte, schmutzigbraune Fließerde, „Grauer Waldboden“

$441 . \mathrm{fBt}$

451 . $\mathrm{fBbt}$ dreigeteilter Eemboden

461 . fBvt 
den an einzelnen Lokalitäten spricht gegen die Auffassung von ZöLLER \& LÖSCHER (1999).

Der letztinterglaziale Bt-Horizont ist im Profil gekappt und wird von einer zweigeteilten Fließerde überlagert (Abschnitt B, Schicht 43). Im unteren Bereich handelt es sich um einen rötlichbraunen sandig-schluffigen Lehm mit feinblättrigem Gefüge und einzelnen eingelagerten Holzkohlen. Auf den Aggregatflächen sind schwache schmutzigbraune Tonüberzüge ausgebildet, die ihrerseits wiederum von hellgrauem Feinstsand überzogen werden. Der obere Abschnitt der Fließerde hat eine graubräunliche Farbe. Hier fehlen die Toncutane, während das feinblättrige Gefüge mit den hellgrauen Feinstsandbelägen wie bei der unterlagernden Schicht entwickelt ist. Die zweigeteilte Fließerde steht in keiner genetischen Beziehung zum liegenden Bt-Horizont und kann deshalb nicht als Al-Horizont gedeutet werden. Dies lässt sich dadurch belegen, dass die Fließerde in einer flachen Mulde den Bt-Horizont kappt. Bei der Fließerde handelt es sich innerhalb des Profils um die erste nachweisbare Abtragungsphase nach dem Eem im Altwürm. Auch Zöller (1995: 75) hat zunächst den beschriebenen Bereich als eine eigenständige Umlagerungszone angesehen. Nach einer späteren Ansicht (vgl. Antoine et al. 2001:217; vgl. auch FrECHEN et al. 2007) soll das Bodensediment von einem interstadialen Grauen Waldboden überprägt worden sein, der an der Oberfläche eine starke Kappung erfahren haben soll. Der Boden wird von ANTOINE et al. (2001: 222) in das Odderade-Interstadial (MIS 5a) gestellt, wofür es in Nußloch jedoch keine sicheren Belege gibt. Im Profil Mainz-Weisenau (BiBus et al. 1996) mit seinem hervorragend gegliederten Altwürmabschnitt wird das Odderade-Interstadial sehr wahrscheinlich durch eine Humuszone repräsentiert, die sich als degradierter Tschernosem deutlich von dem hier diskutierten so genannten Grauen Waldboden unterscheidet. Die Lessivierung im unteren Abschnitt des Bodensediments darf nach unserer Auffassung nicht überinterpretiert werden, da das Ausgangssubstrat aus vorverwittertem und damit vermutlich kalkfreiem Bodensediment bestand.

Über der zweigeteilten Fließerde folgt der Ah-Horizont einer Mosbacher Humuszone (Abschnitt B, Boden WB1, Schicht 42). Die Humuszone ist schwarzbraun gefärbt, weitgehend entkalkt und weist lokal Krotowinen auf. Trotz der Degradation führt sie an einzelnen Stellen Schnecken. Die Humuszone ist im oberen Bereich umgelagert (41), was an einzelnen eingelagerten Steinen erkennbar ist. Zudem sind einzelne Ah-Butzen in den hangenden Löß aufgefroren. Im westlichen Teil des Aufschlusses wird die Humuszone von einem graubräunlichen, geschichteten und nach E einfallenden Sand (36) überlagert. Es handelt sich stratigraphisch bei der Umlagerungszone über der Humuszone und den hangenden Sanden um die stratigraphische Position der Niedereschbacher Zone (vgl. Semmel 1969), die mit erheblichen Diskordanzen verbunden sein kann. Dies zeigt sich auch im östlichen Bereich des Rückens, in dem sich mehrere Rinnen bis in den unteren Bereich des letztinterglazialen Bodens eingeschnitten haben (vgl. Abb. 2). Die westlichste Rinne wird an der Basis von der Fließerde aus Humuszonenmaterial nachgezeichnet (41). Daraus folgt, dass die Einschneidung unmittelbar vor der Entstehung der Fließerde stattgefunden hat. Sie ist anschließend von Sanden und einem grauen Hochflutlehm (38) verfüllt und von abschließenden Sanden überdeckt worden. In der mittleren Rinne lassen sich mehrere Erosionsund Verfüllungsphasen $(37,39)$ nachweisen. Die östlichste Rinne ist hingegen nur von Sand verfüllt worden. Geomorphologisch kann festgehalten werden, dass die Landschaft nach der Verfüllung der Rinnen wieder ihr altes Niveau erreicht hatte. Dennoch sind mit dem beginnenden Mittelwürm, welches mit der Fließerde über der Humuszone einsetzt (Beginn Abschnitt C, Schicht 41), markante landschaftliche Veränderungen abgelaufen (vgl. SEMmEL 1999). In diesem Zusammenhang muss erwähnt werden, dass im östlichsten Teil des Rückens (in Abb. 2 nicht dargestellt) der Altwürmabschnitt vollkommen fehlt. Hier liegt die Niedereschbacher Zone in typischer Ausbildung direkt auf dem 


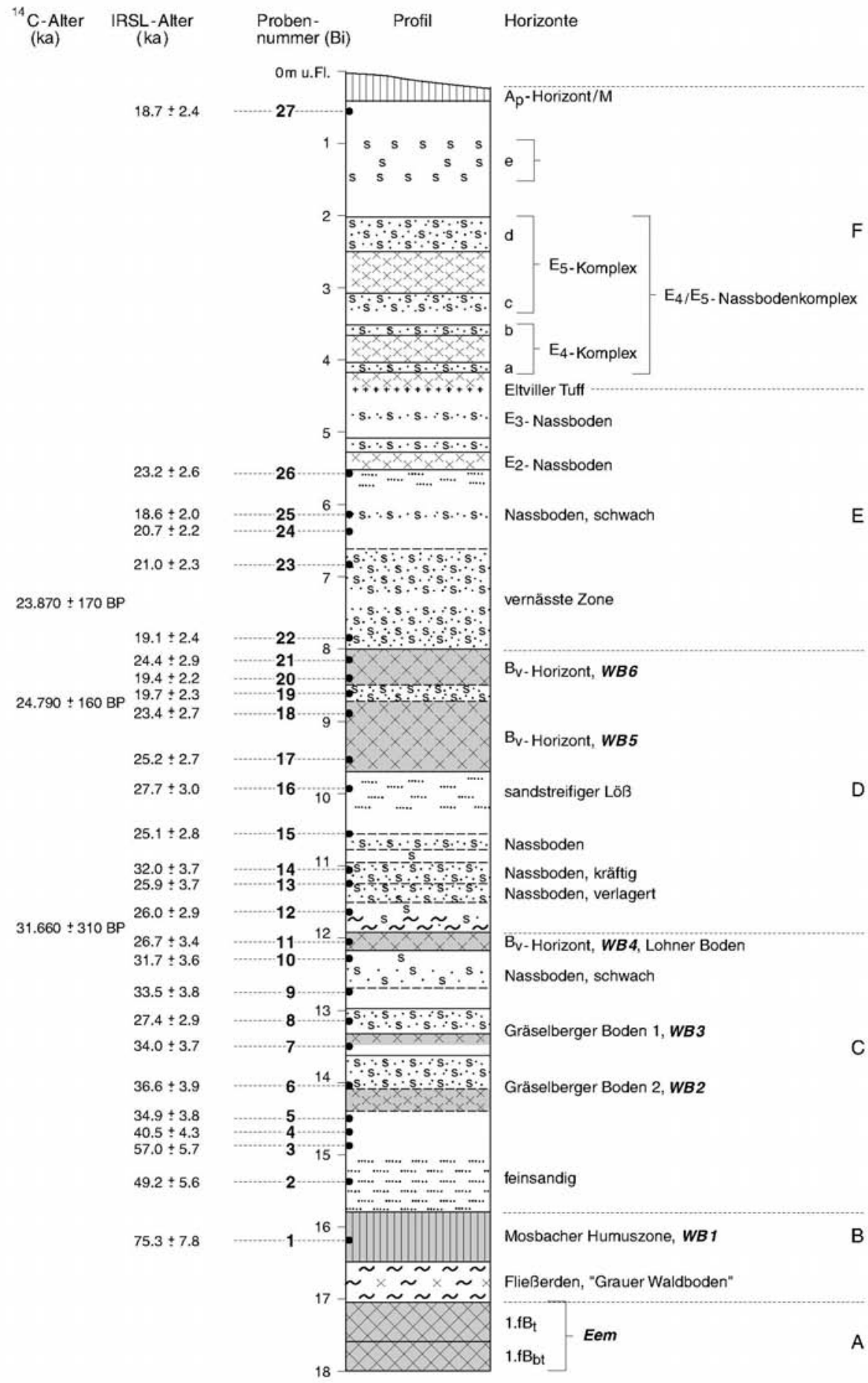

Abb. 3: Standardprofil mit IRSL- und ${ }^{14} \mathrm{C}$-Altern in Nußloch.

Fig.3: Loess/paleosol sequence including radiocarbon and IRSL ages from the Nussloch section. 
letztinterglazialen Bt-Horizont. Zudem ist der Altwürmabschnitt in Nußloch nur teilweise überliefert, da an anderen Lokalitäten noch mindestens zwei weitere interstadiale Humuszonen nachzuweisen sind (vgl. SEMmel 1968, 1996; Bibus et al. 1996).

Über den frühmittelwürmzeitlichen Umlagerungsprodukten setzt Löß ein (Fortsetzung Abschnitt C, Schicht 40), dessen Sedimentation bis in die ausgehende Würmeiszeit anhielt. Im unteren Bereich ist zunächst ein sandiger Löß ausgebildet, der nach oben in typischen Schnecken führenden Löß (35) übergeht. In diesem sind zwei kräftige Nassböden mit z.T. basalen humosen Verbraunungszonen ausgebildet. Der obere Nassboden (WB 3, Schicht 32) ist rostbraun-grau gefleckt, von feinblättriger Struktur und von vielen Fe- und Mn-Knötchen durchsetzt. Lokal können auch rostbraune und graue Bänder ausgebildet sein.

Der untere Nassboden (WB2, Schicht 34) ähnelt sehr stark dem oberen, insgesamt ist er jedoch etwas kräftiger entwickelt. Die Verbraunungszone unter den beiden Nassböden ist schmutzigbraun bis schwach olivstichig gefärbt. Beim oberen Nassboden weist sie auch deutliche Vernässungsmerkmale auf. Kennzeichnend für die beiden Nassböden sowie den Löß unmittelbar darüber sind daumengroße Bleichflecken. Entsprechende Erscheinungen sind von BiBUs (2002) auch in anderen Profilen von Baden-Württemberg beschrieben und als Lauffener Zone (bzw. Nassboden) bezeichnet worden. Die Lauffener Zone ist somit in den basalen Nassbodenkomplex von Nußloch einzuordnen. Stratigraphische Position und Ausprägung der beiden vernässten Böden in Nußloch (WB2 u. WB3) entsprechen dem Gräselberger Boden, der erstmals von SeMmeL (1968) aus dem Rhein-Maingebiet beschrieben wurde. Die Doppelung kann einerseits mit der hohen Sedimentationsrate in Nußloch zusammenhängen; andererseits handelt es sich um einen Abschnitt, der in Lößprofilen sehr selten aufgeschlossen und überliefert ist. $\mathrm{Ob}$ es sich bei den von ZÖLLER (1995) als „Nußlocher Böden“ (zwei Böden in Muldenlage vom Typ arktischer Wiesenböden nach Ansprache von
Van Vliet-LanoË) bezeichneten Horizonten um die hier beschriebenen verbraunten Nassböden handelt, muss offen bleiben, da ZöLLER (1995:73) die Nußlocher Böden älter als die Niedereschbacher Zone einstuft. Die beiden verbraunten Nassböden sowie der gesamte Löß im unteren Profilabschnitt führen reichlich Mollusken, weshalb sich dieser Bereich besonders gut für malakologische Untersuchungen eignet (vgl. Kapitel 5). Der beschriebene Abschnitt ist an anderer Stelle in Nußloch nur durch einen Boden überliefert.

Über dem oberen Gräselberger Boden folgt nur im westlichen Aufschlussbereich eine geringmächtige schwache Nassbodenbildung, die nicht weiter berücksichtigt wird.

Der beschriebene Bereich des Mittelwürmlösses (Abschnitt C) wird von einem kräftigen, braungefärbten Bv-Horizont (Boden WB4, Schicht 31) abgeschlossen, der neben der Mosbacher Humuszone die markanteste Bodenbildung innerhalb des Würmlösses darstellt. Es handelt sich um einen schwach rötlichbraunen, lehmigen Schluff mit sehr vielen $\mathrm{Mn}$ - und Fe-Knötchen und -Nadelstichen im oberen Bereich. In dem kalkhaltigen Horizont sind viele Schnecken eingelagert. Er weist an einzelnen Stellen eine feine Frostblättrigkeit oder eine wellig-plattige Struktur mit schwachen Tonanflügen auf. Makroskopisch wie mikromorphologisch lassen sich keine Hinweise auf stärkere Umlagerung feststellen. Die wechselnde Struktur kann jedoch ein Hinweis auf lokale schwache Umlagerung sein. Die Durchgängigkeit des Horizontes, auch in anderen Aufschlussbereichen, spricht für eine eigenständige Bodenbildung. Aufgrund der deutlichen Verbraunung aber nur mäßigen Entkalkung und Verlehmung muss der Horizont typologisch als ein Übergang zwischen einem $\mathrm{Cv}$ - und $\mathrm{Bv}$-Horizont angesehen werden. Aus Gründen der Vereinfachung werden im Folgenden verbraunte Horizonte auch ohne Verlehmung als Bv-Horizonte angesprochen. Die stratigraphische Einstufung des Bv-Horizontes bereitet auf Grund seiner tiefen Lage im Profil sowie seiner recht kräftigen Ausbildung Schwierigkeiten. Da der Boden aber lokal 
die typischen Merkmale des Lohners Bodens besitzt (rostige Mn-und Fe-Nadelstiche, FeKnötchen, welligplattige Struktur, Tonanflüge, schwache diffuse Mn-Ausfällungen), wurde er letztendlich mit dem Lohner Boden parallelisiert. Auch eine Korrelation mit dem Böckinger Boden oder einer älteren mittelwürmzeitlichen Bildung wäre nicht auszuschließen. Dagegen sprechen jedoch die durchgeführten absoluten Datierungen (vgl. Kapitel 6).

Unmittelbar über dem als Lohner Boden angesehenen Bv-Horizont (WB4) liegt eine vernässte Umlagerungszone (Beginn Bereich Abschnitt D, Schicht 30), die mit dem unterlagernden Boden in keiner genetischen Beziehung steht. Es handelt sich um einen Nassboden, der von BiBus (2002) als E0-Nassboden bezeichnet wird. Im darüber liegenden Löß folgen drei weitere Nassböden $(29,28,27)$, die im Zentrum des Rückens klar durch Löß voneinander getrennt sind, im Randbereich jedoch zusammenlaufen und dann nur einen schlecht zu gliedernden Nassbodenkomplex bilden.

Der oberste Nassboden (27) ist am schwächsten ausgebildet und zudem sehr stark verflossen. Es handelt sich um eine vernässte Zone, in der die Bleichflecken schrägt gestellt sind. Lokal wurde unter diesem Nassboden ein sehr schwacher Ah-Horizont beobachtet.

Zwischen dem mittleren und dem unteren Nassboden sowie über dem oberen Nassboden ist der Löß (z.B. Schicht 26) aufgrund von Flugsandeinfluss stark sandstreifig ausgebildet. Nach oben abgeschlossen wird der beschriebene Abschnitt durch einen Bodenkomplex, der in der Regel durch zwei Nassböden mit einem jeweils darunter liegenden, undeutlichen $\mathrm{Bv}-$ Horizont zweigegliedert ist. Da an einer Stelle der untere Boden nochmals untergliedert werden konnte, muss sogar mit einer Dreiteilung gerechnet werden. Bei den Aufnahmen und Untersuchungen wurde diese Stelle jedoch nicht berücksichtigt.

Die beiden Böden sind sehr ähnlich ausgebildet. Der obere Bv-Horizont (Boden WB6, Schicht 23) wird von einer sehr kräftigen Vernässungszone (Ø $80 \mathrm{~cm}$, Schicht 22) überlagert. Es handelt sich um einen fahlgrauen, feinsandigen
Schluff mit intensiven Rostflecken, -schlieren und -knötchen. Der Bv-Horizont des Bodens (Ø $50 \mathrm{~cm}$, Schicht 25) ist schwach braun gefärbt und rostfleckig, hat eine wellig-blättrige Struktur mit Fe- und Tonanflügen und weist an der Obergrenze diffuse Mn-Flecken auf.

Der darunter liegende Bv-Horizont (Boden WB5, $\varnothing$ bis $80 \mathrm{~cm}$ ) ist mit einer olivbräunlichen Farbe kräftiger ausgebildet als der obere $\mathrm{Bv}$-Horizont. Er ist etwas dichter gelagert und nur schwach frostblättrig. Typisch sind im oberen Bereich ebenfalls leuchtend rote Rostknötchen und diffuse Mn-Flecken. Die Vernässungszone über dem Boden (24) ist als fahlgrauer feinsandiger Schluff mit intensiven Fe- und Mn-Flecken kräftig ausgebildet.

Am Anfang unserer Untersuchungen hatten wir den oberen Bv-Horizont als den Lohner und den unteren Bv-Horizont als den Böckinger Boden angesehen. Von dieser Vorstellung sind wir aber deswegen abgekommen, weil die beiden Böden sehr schwach ausgebildet sind und sie eher eine feinblättrige statt eine wellig-plattige Struktur besitzen, wie dies beim Lohner und Böckinger Boden der Fall ist. Zudem haben die absoluten Datierungen jungwürmzeitliche Alter erbracht (vgl. Kapitel 6).

Ungefähr $90 \mathrm{~cm}$ über der vernässten Umlagerungszone des oberen $\mathrm{Bv}$-Horizontes (Abschnitt E) ist in einem gelblichen Löß (21) ein geringmächtiger Nassboden $(\varnothing 10 \mathrm{~cm}$, Schicht 20) ausgebildet, der sich durch einzelne intensive Rostflecken und nach unten geöffnete Rosthalbmonde auszeichnet.

Im Hangenden folgt sandstreifiger Löß (19, 18) und ein weiterer, nunmehr sehr kräftiger, z.T. zweiteiliger Nassboden (16). Mächtigkeit und Intensität des Horizontes verändern sich in Nußloch sehr stark innerhalb des Aufschlusses. Bei schwacher Ausbildung ist er nur $20 \mathrm{~cm}$ mächtig (fahlrötlich-grauer Vernässungshorizont mit daumengroßen Rostflecken, z.T. halbmondartig, darunter schwach gelblichbrauner $\mathrm{Bv}$, oben und unten vernässte Randsäume). Im östlichen Bereich des Aufschlusses liegt unter dem Nassboden umgelagertes, kräftig braun gefärbtes Material (17), welches offensichtlich aus einem Bt-Horizont stammt. Das Bodense- 
diment ist durch Fließvorgänge im Zuge der Nassbodenbildung entstanden. Bemerkenswerterweise war auch in einem anderen Aufschlussbereich in gleicher stratigraphischer Position entsprechendes rötlichbraunes Bodensediment nachzuweisen. Der beschriebene Bereich liegt zweifelfrei im Abschnitt des E2Nassbodens.

Darüber tritt im Löß (15) eine schwache Vernässung (14) auf, die als E3- Nassboden angesehen wird. Eine solche Zuordnung kann deshalb sicher erfolgen, weil unmittelbar im Hangenden der Eltviller Tuff (13) vorhanden ist. Das schwarzgraue, wellig verlaufende Tuffband $(\varnothing$ $0,5-1 \mathrm{~cm})$ ist lokal zwei- oder dreigeteilt. Auf kurze Entfernung kann es in Flecken aufgelöst sein oder auch fehlen. Mit einem Alter von 19,5 - 20,6 ka (Altersmittelwert n. FreCHEN \& Preusser 1996: 63, vgl. auch Antoine et al. 2001: 226) stellt der Eltviller Tuff die wichtigste, stratigraphisch sicher zuzuordnende Leitschicht innerhalb des Jungwürmlösses dar. Ohne ihn wäre in Nußloch aufgrund der differenzierten Ausbildung des obersten Jungwürmabschnitts eine sichere Zuordnung kaum möglich.

Über dem Eltviller Tuff liegt ein mehrere Meter mächtiger Nassbodenkomplex (Abschnitt F). Insgesamt lassen sich mindestens fünf Vernässungszonen mit z.T. schwachen unterlagernden Verbaunungshorizonten ausweisen. Die beiden oberen Nassböden sind am schwächsten ausgebildet. Der Eltviller Tuff liegt an der Untergrenze des untersten Bv-Horizontes (12). Dieser Boden entspricht einschließlich der hangenden Vernässungszone dem E4-Nassboden der Erbenheimer Böden i.S.v. Semmel (1968). Ob jedoch der nächst jüngere Nassboden aufgrund von starker Sedimentation und Spreizung auch noch zum E4 gehört oder bereits dem E5 zugeordnet werden muss, ist schwer zu entscheiden, zumal der E5-Nassboden sehr selten aufgeschlossen ist. ZöLLER \& LÖSCHER (1999: 49) haben in einem anderen Profil in Nußloch den „Maisbacher Boden“ als schwachen Bv-Horizont direkt zwischen einem als E4 und E5 angesehenen Nassboden ausgegliedert. Aufgrund des vorliegenden Profils mit wesentlich mehr Vernässungszonen und Bv-Horizonten kann diese Interpretation nicht mehr aufrecht erhalten werden. Es soll deshalb im neu bearbeiteten Profil von einem E4/E5-Nassbodenkomplex gesprochen werden, wobei die einzelnen Nassböden mit a, b, c, d und e von unten nach oben durchnummeriert wurden (vgl. Abb. 2 u. 3). Da der gesamte Nassbodenkomplex auf stark sandigem Löß ausgebildet ist, erhebt sich für die jeweils im Liegenden der Nassböden ausgebildeten, sehr schwachen Bv-Horizonte die Frage, ob sie überhaupt pedogen sind und nicht durch das Substrat vorgetäuscht werden. Auch über dem E4/E5-Nassbodenkomplex ist der Löß (4) lokal schwach verbraunt und mit einzelnen Rostschlieren durchsetzt. Nur im östlichen Aufschlussbereich folgt als Abschluss ein jüngster graugelber Löß, auf dem noch lokal Reste des rezenten Bt-Horizontes erhalten sind. Im übrigen Aufschluss liegt als Folge der Bodenerosion eine Kultopararendzina vor.

Als Fazit bei der Interpretation des Würmlösses in Nußloch stellt sich das Problem, welcher der beschriebenen Verbraunungshorizonte dem Lohner Boden entspricht.

Wenn der Bv-Horizont (WB4) im unteren Profilabschnitt, wie wir es zuletzt getan haben, als Lohner Boden angesehen wird, dann ergibt sich die Schwierigkeit, dass der bis $8 \mathrm{~m}$ mächtige Abschnitt zwischen dem E3-Nassboden (bzw. dem Eltviller Tuff) und dem unteren kräftigen Bv- Horizont (WB4) dem Abschnitt E0 bis E2 entspricht, was aufgrund der großen Mächtigkeit doch sehr überrascht.

Würde der Bodenkomplex WB5/WB6 mit dem Lohner Boden und dem Böckinger Boden parallelisiert, dann könnte der hangende Löß mit den darin enthaltenen Nassböden relativ problemlos mit dem älteren Jungwürmabschnitt (E0, E1, E2) und der darunter liegende Löß mit dem Mittelwürmlöß und den darin vorkommenden Nassböden parallelisiert werden. Es ergäbe sich dabei allerdings die Frage, um welchen Boden es sich bei dem intensiv braunen Bv-Horizont (WB4) im unteren Profilabschnitt handelt. Er müsste dann einem älteren, bislang nicht bekannten, würminterstadialen Boden entsprechen. 


\section{Sedimentuntersuchungen im Profil Nußloch}

Im sedimentologisch untersuchten Profil (vgl. Abb.4) tritt der höchste Tongehalt mit ca. $32 \%$ im oberen Bereich des letztinterglazialen BtHorizontes auf. Zur Untergrenze sinken die Werte kontinuierlich bis auf einen Tongehalt von ca. $13 \%$ ab. Die Korngrößenanalysen liefern somit kein Indiz für eine polygenetische Bildung mit mehreren Bt-Horizonten.

Die den letztinterglazialen Bt-Horizont überlagernde zweigliedrige Fließerde, der so genannte „Graue Waldboden“, weist, abgesehen von einer im Profil höher liegenden Sandlage, mit ca. $20 \%$ den höchsten Sandgehalt (Durchschnitt ca. $5 \%$ ) auf. Dieser Sachverhalt könnte ein Hinweis dafür sein, dass es sich um ein umgelagertes Bodensediment handelt.

Die Mosbacher Humuszone (Boden WB1) besitzt mit 0,5\% einen relativ geringen Anteil an Kohlenstoff. Dieser Wert wird auch noch im Hangenden bei weiteren innerwürmzeitlichen Bodenbildungen erreicht. Oberhalb der Humuszone setzt an der Grenze vom Alt- zum Mittelwürm (Grenze Abschnitt B/C) der Kalkgehalt ein, der mit ca. $15-35 \%$ eine relativ große Schwankungsbreite besitzt.

An der Basis des Mittelwürmprofils sind im Bereich der Niedereschbacher Zone zum Teil mit Sand verfüllte Rinnen vorhanden. Auch im hangenden Löß tritt ein deutlicher Sandanteil auf, der auf Flugsandbeimengungen zurückgeführt werden kann. Der Sandanteil liegt ansonsten, bis auf zwei Ausnahmen, im gesamten Würmlöß unter $5 \%$.

Im Abschnitt mit den Gräselberger Böden (WB2 und WB3) steigt die organische Substanz geringfügig an. Dieser Befund stimmt mit der etwas dunkleren Färbung der beiden Böden und dem dazwischen liegenden Löß überein.

Innerhalb der Laboranalysen sticht der kräftige Verbraunungshorizont (WB4), der mit dem Lohner Boden parallelisiert wurde, deutlich heraus. Er weist einen höheren Tongehalt $(12,8 \%)$ und geringere $\mathrm{CaCO}_{3}$-Anteile als der hangende und liegende Löß auf. Zudem besitzt er mit $0,6 \%$ den höchsten Anteil an organischer Substanz im gesamten Profil.

Im Jungwürmlöß sind bei den Laboranalysen keine Besonderheiten zu beobachten. Nur im Bereich der zwei schwachen verbraunten Nassböden (WB5, WB6) sowie in einem Abschnitt des E4/E5-Nassbodenkomplexes wurde ein geringfügig höherer Sandanteil registriert. Eine Sandstreifigkeit dieser Abschnitte wurde auch bei der Profilaufnahme festgestellt.

Das Standardprofil wurde lückenlos geophysikalisch untersucht, indem an 951 Proben die magnetische Suszeptibilität im Labor gemessen worden ist (vgl. Abb. 5). Die Suszeptibilität zeigt den Gehalt des ferrimagnetischen Materials sowie den Anteil der superparamagnetischen Bestandteile in einer Probe an. Durch pedogene Prozesse werden diese Minerale, vor allem die weichmagnetischen Teilchen, erhöht (vgl. HeLLER \& Evans 1995), so dass die Suszeptibilität ein Parameter für Verwitterungsvorgänge und -intensität und damit für Klimaschwankungen ist. Die Bildung von pedogenen magnetischen Mineralen wird in günstigeren Klimaabschnitten durch anorganische (MAHER \& THOMPSON 1995) und organische (z .B. bakteriologische Aktivitäten) Prozesse erklärt (vgl. FASSBINDER et al. 1990), wobei über die einzelnen Vorgänge im Boden wohl noch Unklarheit herrscht (vgl. auch Meng et al. 1997).

Die Suszeptibilitätskurve weist im Profil Nußloch einen wechselnden Verlauf mit z.T. starken Schwankungen auf. Es lassen sich drei Abschnitte ausgliedern.

Im oberen Abschnitt bis zur Obergrenze des Bodens WB6 (ca. $8 \mathrm{~m} \mathrm{u}$. Fl.) sind die Schwankungen gering und bewegen sich auf einem niedrigen Niveau. Es ist der Bereich der Löß- und Nassbodensequenzen im oberen Jungwürm. Eine typische Zuordnung der Suszeptibilität zu Nassböden ist nicht möglich. Nur der rezente Ap-Horizont sowie der Eltviller Tuff stechen durch starke Peaks heraus. Ab $8 \mathrm{~m}$ Profiltiefe mit der Obergrenze des Bodens WB6 erhöht sich die Suszeptibilität deutlich, aber auch hier sind die Schwankungen nicht signifikant. Die beiden Böden WB5 und WB6 zeichnen sich in der Kurve nicht ab. Anders 


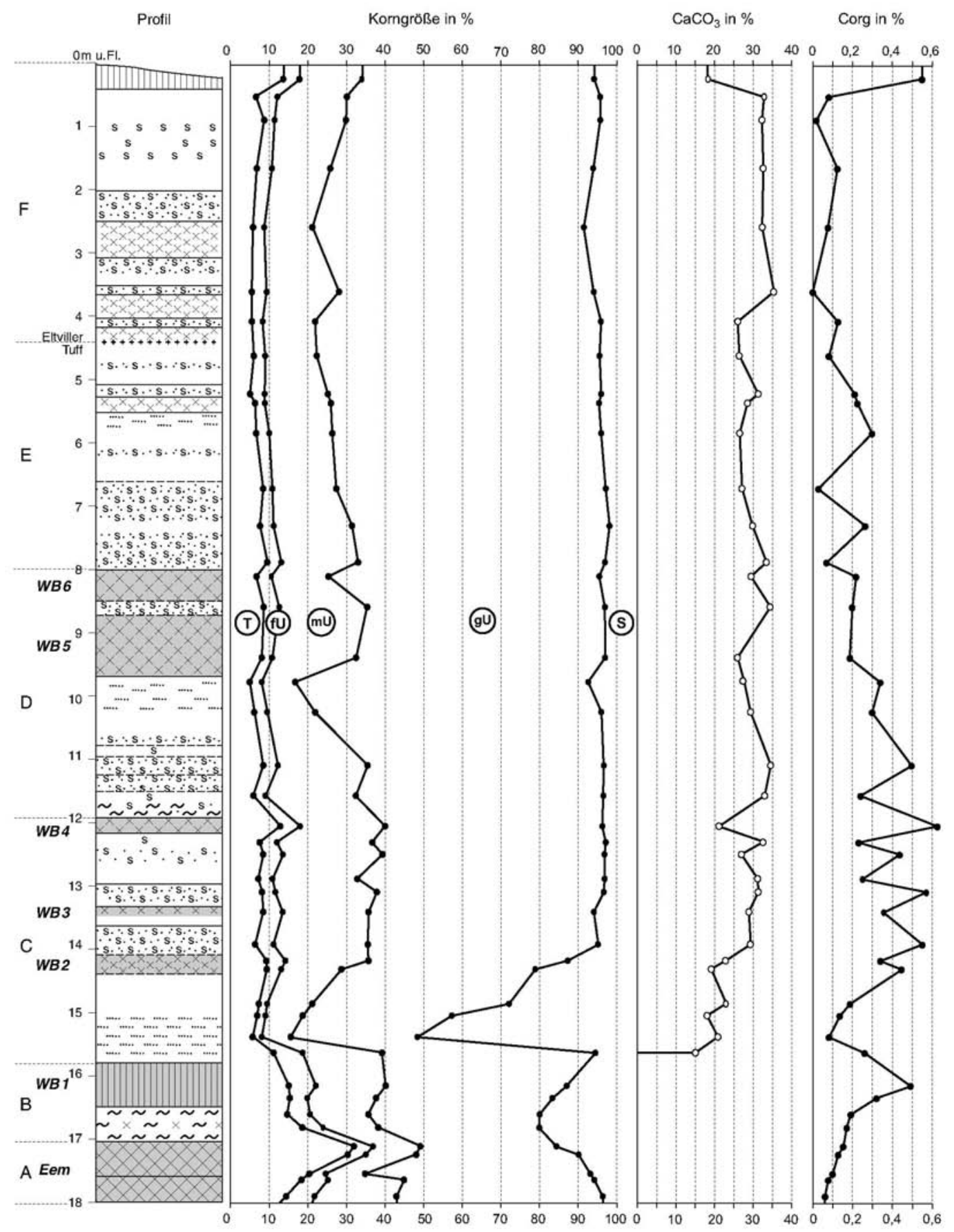

Abb. 4: Sedimentanalysen des untersuchten Standardprofils in Nußloch.

Fig. 4: Loess/paleosol sequence including sedimentological results from the Nussloch section. 


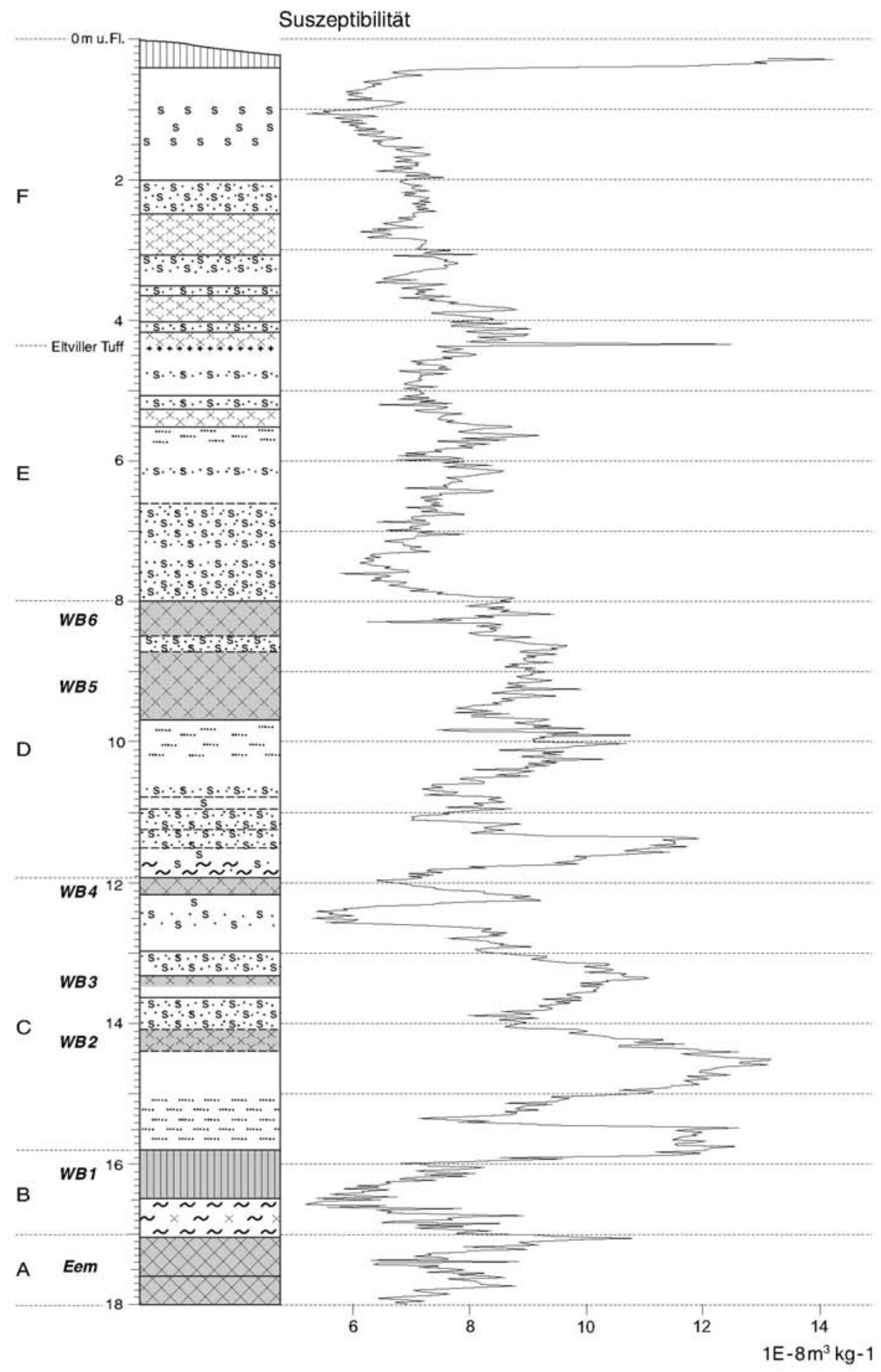

Abb. 5: Kurve der magnetischen Suszeptibilität im Jungpleistozän von Nußloch.

Fig. 5: Loess/paleosol sequence including results of magnetic susceptibility measurements from the Nussloch section. 
wird die Situation ab 11,50 m unter Flur bis in den Bereich des Eembodens. Hier schwankt die Suszeptibilität sehr stark, wobei die Spitzen eindeutig den ausgegliederten interstadialen Böden des Mittelwürms zugeordnet werden können. Interessant ist dabei, dass die kräftigen Böden, wie z. B. der Lohner Boden (WB4), die Mosbacher Humuszone (WB1) und der letztinterglaziale Boden nicht die höchsten Werte besitzen. Für den letztinterglazialen Boden könnten wie in Mainz-Weisenau (vgl. BiBus et al. 1996) oder in Schatthausen (TerHORST et. al. 2001) sekundäre Staunässeeinflüsse mit Lösung ferrimagnetischer Bestandteile angenommen werden, zumal häufig Sesquioxidkonkretionen im Solummaterial auftreten. Doch sind die Pseudovergleyungsmerkmale insgesamt gering und die Suszeptibilitätswerte an der Obergrenze des Bodens am höchsten. Schwer zu erklären ist weiterhin, warum der z.T. verlagerte Nassboden (11,50 m unter Flur) über dem Lohner Boden (WB4) einen Spitzenwert bei der Suszeptibilität besitzt, da sich die Nassböden doch zumeist durch niedrige Werte auszeichnen. Eine Erklärung wäre es, dass in diesem Abschnitt vulkanisches Material des Rambacher Tuffs aufgearbeitet wurde, der in hessischen Profilen dicht über dem Lohner Boden gefunden worden ist (SEMmel 1967; Bibus 1973: 351).

Für das stark durch Diskordanzen geprägte Profil Schatthausen haben Terhorst et al. (2001) bei gesteinsmagnetischen Untersuchungen zeigen können, dass die zwischen Mosbacher Humuszone und Eemboden liegende, schwach lessivierte Verlagerungszone (so genannter Grauer Waldboden) im mittleren und unteren Teil die höchsten Suszeptibilitätswerte besitzt. Im Profil Nußloch können wir diese Ergebnisse nicht bestätigen. Übereinstimmung herrscht dagegen in dem Befund, dass im oberen Bereich des Grauen Waldbodens (EBt Horizont nach Terhorst et al. 2001) mit die niedrigsten Suszeptibilitätswerte in der Eem-/ Würmabfolge auftreten. Eine an anderer Stelle von ANTOINE et al. (2001) in Nußloch erstellte Suszeptibilitätskurve lässt sich nur schwer mit unseren Befunden parallelisieren, zumal die stratigraphischen Korrelationen zwischen den Profilen nicht sicher sind. Insgesamt lässt sich jedoch in dem von uns untersuchten Profil zeigen, dass gesteinsmagnetische Parameter eine hilfreiche Bestätigung der im Gelände erkannten schwächeren Paläoböden sein können. Es fehlen jedoch systematische Untersuchungen über unterschiedliche Lösse, Paläoböden und Klimaprovinzen. Es kann deshalb auch bezweifelt werden, dass es künftig in Mitteleuropa möglich sein wird, anhand der Suszeptibilität quantitative Klimaaussagen zu machen, wie dies in chinesischen Lössen versucht worden ist (Heller et al. 1993; MAHER et al. 1994; MAHER \& THOMPSON 1995; Florindo et al. 1999). Im Profil Nußloch wurden außerdem in ausgewählten Abschnitten paläomagnetische Messungen mit dem Ziel durchgeführt, den in Sedimenten des Nordatlantiks nachgewiesenen Mono Lake- und den Laschamp-Event zu finden (LAJ et al. 2000). Leider haben diese Untersuchungen keine positiven Ergebnisse erbracht.

\section{Biomorphologische Ergebnisse im Profil Nußloch}

An 25 Proben aus dem Profil wurden von A. Goljewa (Geogr. Institut, Moskau, schriftl. Mitteilung) biomorphologische Untersuchungen durchgeführt. Es sind die oberen Bereiche der unterschiedlichen Paläoböden sowie die unmittelbar hangenden Sedimente beprobt worden. Da für eine statistische Auswertung keine genügende Anzahl von biomorphen Bestandteilen vorhanden war, können nur qualitative und halbquantitative Aussagen gemacht werden. Es muss zudem berücksichtigt werden, dass die ursprüngliche Oberfläche der Böden zumeist gekappt wurde, was für die biomorphologischen Untersuchungen nachteilig ist. Im obersten, bereits mit Lößbutzen durchsetzten Bereich der Mosbacher Humuszone (Boden WB1) treten Detritus und Opalphytolithe auf, bei denen die Wiesengräser (Süßgräser, Riedgräser) dominieren. Es muss von einer unterschiedlich zusammengesetzten Wiesenvegetation zum Ende der Schwarzerdebildung 
ausgegangen werden. Im überlagernden Löß sind keine Phytolithe enthalten. Im oberen Bereich des Ah-Horizontes ist eine ähnliche Zusammensetzung von Detritus und Phytolithen wie im darüber folgenden ,Vermischungshorizont" vorhanden, doch nimmt die Anzahl der Phytolithe ab. In der Mitte der Mosbacher Humuszone konnten hingegen keine Phytolithe nachgewiesen werden. Dies könnte für eine länger anhaltende Schwarzerdebildung ohne in die Tiefe gehende Vermischungsprozesse sprechen, was allerdings den Vorstellungen einer Schwarzerdebildung widerspricht. Die zweigeteilte Fließerde zwischen der Mosbacher Humuszone und dem letztinterglazialen Bt-Horizont, der so genannte „Graue Waldboden", führt im obersten, noch von der Humuszone überprägten Abschnitt viel Detritus und Phytolithe. Mit abnehmender Häufigkeit treten Phytolithe von Moosen, Farnen, Wiesengräsern und vereinzelt von Waldgräsern auf. Es ergibt sich damit das Vegetationsbild eines lichten Laubwaldes. Eine tiefer liegende Probe aus dem „Grauen Waldboden“ erbrachte eine ähnliche Phytolith-Zusammensetzung. Allerdings konnten hier keine Waldgräser-Phytolithe nachgewiesen werden, was für eine offene Vegetation spricht. Diese Befunde zeigen, wie auch die Horizontabfolge, einen komplexeren Aufbau des basalen Würmabschnitts. Die Ergebnisse stehen etwas im Widerspruch zu der Tatsache, dass in dem „Grauen Waldboden“ in anderen Profilen oft Holzkohlebröckchen von Koniferen gefunden wurden. FRECHEN et al. (2007) führen diese in dem benachbarten Profil Schatthausen auf Umlagerung zurück und diskutieren ausführlich die Komplexität des Altwürmabschnitts. Eine Probe aus dem kräftigen, als Lohner Boden angesprochenem Bv-Horizont (Boden WB4) führte wenige Phytolithe, vor allem von Wiesengräsern, während Waldformen nicht nachzuweisen waren.

Bei den beiden schwach verbraunten Nassböden im mittleren Profilabschnitt (Böden WB5 und WB6) führen die vernässten Horizonte keine Phytolithe. In dem oberen Bereich der beiden Bv-Horizonte treten wenige, nicht näher zu identifizierende Phytolithe auf. Of- fensichtlich waren Wald-, Wiesen- und Riedgräser abwesend. Wahrscheinlich war eine Tundrenvegetation ausgebildet. Anders sind jedoch die Verhältnisse an der Obergrenze des Bv-Horizontes vom E4-Nassbodens. Es treten in etwa mit gleichen Anteilen Phytolithe von Wiesen-, Wald- und Riedgräsern sowie Moosen und Dikotyledonen auf, die zu einem lichten Laubwald mit gut entwickelter Gras- und Moosschicht passen könnten.

Die biomorphologischen Untersuchungen durch GolJewa haben gezeigt, dass in den untersuchten Vernässungszonen von Nassböden sowie im reinen Löß keine Opalphytolithe vorhanden sind. Die kräftigen würmzeitlichen Böden haben hingegen, obwohl sie z.T. oberflächlich etwas erodiert sind, Hinweise auf die zur Bodenbildungszeit vorhandene Vegetation gegeben, wobei Differenzierungen möglich sind.

\section{Malakozoologische Befunde in Nußloch}

\subsection{Molluskenführung}

Für das Studium der Molluskenfaunen wurde der Mittel- und Jungwürmlöß des in dieser Arbeit beschriebenen Profils horizontiert, aber nicht immer in regelmäßigen Abständen, an 63 Stellen beprobt. Die Entnahmestellen der Proben sind der Abbildung 6 zu entnehmen. Weitere Proben enthielten entweder nur schlecht erhaltenes, nicht näher identifizierbares Material (Mosbacher Humuszone) oder überhaupt keine Fossilien (Abschnitte c-e des E4/E5-Nassbodenkomplexes).

Das Schalenmaterial in den jeweils 8 Liter umfassenden Sedimentproben wurde nach dem Schlämmen (Maschenweite der Siebe: 0,8 mm) unter dem Stereomikroskop quantitativ ausgelesen und bestimmt. Anschließend wurde nach den üblichen Methoden für die einzelnen Arten die Mindestzahl der in einer Probe vorhandenen Individuen ermittelt. Für die Wiedergabe der Häufigkeit der einzelnen Arten sowie der Summe der in einer Probe festgestellten Individuen wurde in Abbildung 6 eine duallogarithmische Darstellung gewählt.

Insgesamt konnten Reste von 22 Schnecken- 


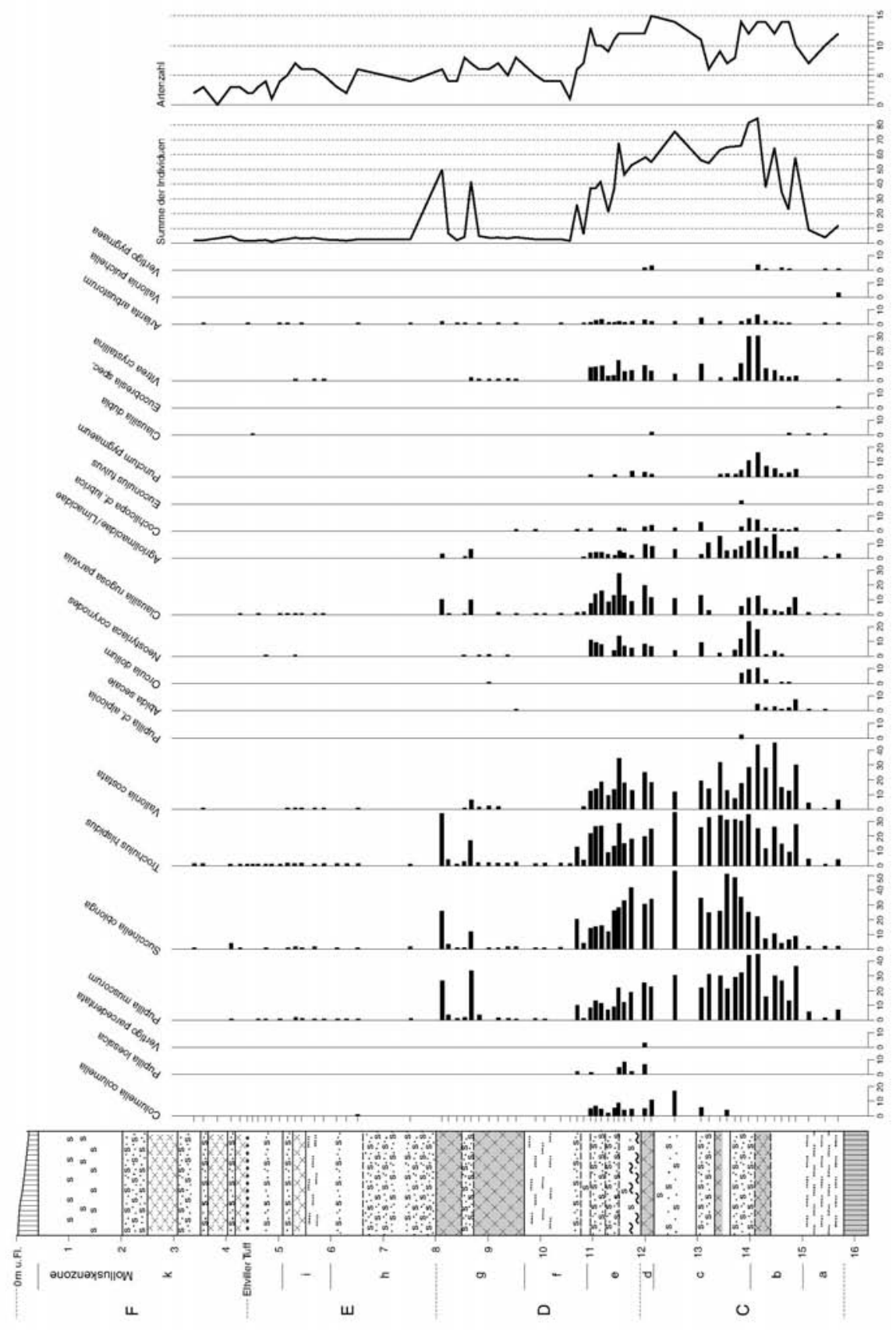

Abb. 6: Struktur der Molluskenfaunen im jungpleistozänen Lößprofil von Nußloch. Wiedergabe der Häufigkeit der Arten in duallogarithmischer Darstellung, d.h. die angegebenen Werte entsprechen den Quadratwurzeln der tatsächlich festgestellten Individuenzahlen.

Fig. 6: Mollusc assemblages in the wuermian loess profile of Nussloch. The abundance of the species in the samples is expressed by the square roots of the really counted individuals. 
arten nachgewiesen werden. Maximal waren in einer Probe 15 Arten zu finden. Besonders zahlreich und zuweilen geradezu massenhaft traten Schnecken im unteren Drittel der Abfolge auf. Dagegen war im jüngeren Löss, von zwei Horizonten einmal abgesehen, die Ausbeute eher spärlich. Selten konnten dort mehr als zehn Individuen in einer Sedimentprobe registriert werden.

Die Faunen setzen sich aus Offenlandarten sowie anpassungsfähigen Ubiquisten zusammen und bezeugen für sämtliche Abschnitte des Profils eine Landschaft ohne höhere Vegetation. Den Grundstock der Faunen bilden die kaltzeitlichen Häufigkeitsarten Pupilla muscorum, Succinella oblonga und Trochulus hispidus (früher: Trichia hispida). Sie sind in den allermeisten Horizonten zu finden, und ihr Anteil an der Gesamtzahl der ausgelesenen Individuen beträgt selten weniger als $50 \%$. Häufig entfallen sogar mehr als $80 \%$ auf diese drei Arten.

Eine Differenzierung erfahren die Lößfaunen in erster Linie durch Begleitarten, deren Auftauchen oder Verschwinden Rückschlüsse auf klimaökologische Veränderungen zulässt. Hier sind an erster Stelle die hoch kaltzeitlichen Indexarten Columella columella, Vertigo parcedentata und Pupilla loessica zu nennen, sowie einige Arten, die zwar vorwiegend in Warmzeiten auftreten, aber auch in milderen Abschnitten von Kaltzeiten anwesend sein können (Vertigo pygmaea, Vallonia pulchella). Die mit Interstadialen verbundenen wärmeren Schwankungen waren zu schwach oder zu kurz, um gravierende Veränderung in der Malakofauna, geschweige denn eine Auslöschung der etablierten kaltzeitlichen Fauna zu bewirken. Deshalb ist das vielfach zu beobachtende Nebeneinander von hoch kaltzeitlichen Elementen und klimatisch anspruchsvolleren Arten geradezu charakteristisch für Molluskenfaunen aus Interstadialen. Besondere Aufmerksamkeit verdient in diesem Zusammenhang auch die Anwesenheit alpiner Faunenelemente wie Orcula dolium oder Neostyriaca corynodes in Teilen des Profiles, sowie das Auftreten von Abida secale (s. u.). Schlussfolgerungen klimaökologischer Art lassen sich unter Umständen auch aus der Armut oder dem
Reichtum an Arten oder aus den unterschiedlich hohen Individuenzahlen in den einzelnen Proben ziehen.

\subsection{Paläoökologische Analyse der Molluskenfaunen}

Mit Hilfe der Molluskenfaunen und deren $\mathrm{Zu}$ sammensetzung lässt sich das vorliegende Lößprofil in mindestens 10 Zonen einteilen (Abb. 6, Molluskenzonen a - k). Was die Abgrenzung dieser Zonen angeht, so sind nicht immer, aber doch in den meisten Fällen deutliche Beziehungen zur paläopedologischen Gliederung der Abfolge in die Abschnitte B bis F (Abb. 3) zu erkennen.

Zone a: Niedereschbacher Zone

In diesem frühen mittelwürmzeitlichen $\mathrm{Ab}$ schnitt wurden insgesamt 14 Schneckenarten festgestellt. Die Individuenzahlen sind erheblich geringer als in dem darüber einsetzenden Löß. Die Anwesenheit von klimaempfindlicheren Faunenelementen wie Vertigo pygmaea und Vallonia pulchella lassen auf einen eher milden Abschnitt der Kaltzeit schließen.

\section{Zone b: Mittelwürmlöß einschließlich Verbraunungszone an der Basis des Nassbodens WB2}

Besonders bezeichnend für diesen Profilabschnitt ist das regelmäßige Vorkommen von Abida secale, einer alpin-südwesteuropäischen Art. Sie tritt in der gesamten Abfolge nur noch einmal im oberen Bereich des Profils (WB5) auf. Die ökologische Amplitude von A. secale ist weit gespannt. Die Kalk liebende Schnecke lebt sowohl in lichten Wäldern als auch an Felsen und auf trockenen Rasenflächen. Im höheren Bergland verhält sie sich deutlich thermophil. Insgesamt ist anzunehmen, dass $A$. secale zu den anspruchsvollsten Arten aus dem Nußlocher Lößprofil zählt und als Indexart für mildere Abschnitte der Kaltzeit den schon genannten Formen Vertigo pygmaea und Vallonia pulchella an die Seite zu stellen ist. 
Neben dem gelegentlichen Auftreten des klimaempfindlichen Vertigo pygmaea fällt in diesem Profilabschnitt die Häufigkeit von Schnecken mit geringem Feuchtigkeitsbedürfnis auf (u. a. Pupilla muscorum, Vallonia costata, Clausilia rugosa parvula). Ihr Anteil kann bei den Individuenzahlen zuweilen mehr als $75 \%$ erreichen (WB2). Vallonia costata, eine Art, die zwar nicht gerade $\mathrm{zu}$ den klimatisch anspruchsvollsten Schnecken gehört, aber deutlich die milderen Phasen der Kaltzeiten bevorzugt (Rousseau 1989), ist fast überall reichlich und zuweilen geradezu massenhaft zu finden. Außerordentlich gering sind dagegen die Anteile der hygrophilen Lößschnecke Succinella oblonga.

Insgesamt entspricht die Zusammensetzung der Schneckenfauna einem relativ milden und überwiegend trockenen Abschnitt des Mittelwürms. Erst gegen Ende dieser Phase (im Übergangsbereich zu Molluskenzone c) ist eine allmähliche Verbesserung des Feuchtigkeitsangebotes festzustellen. An dieser Stelle wurden die mit Abstand höchsten Individuenzahlen des gesamten Profiles registriert. Die Feuchte liebende Art Vitrea crystallina erreicht hier mit Anteilen von mehr als $13 \%$ besonders hohe Abundanzwerte (Abb. 6).

\section{Zone c: Nassböden und Löße im Liegenden des Bv-Horizontes WB4}

Die Individuenzahlen in diesem Profilabschnitt sind nach wie vor hoch. Es deutet sich jedoch mit dem Verschwinden anspruchsvollerer Arten (Vertigo pygmaea, Abida secale) sowie in der deutlichen Abnahme der Artenzahlen eine Klimaverschärfung an, die in der Zeit zwischen der Bildung der beiden Nassböden WB2 und WB3 einen ersten Höhepunkt erreicht zu haben scheint. Hier tritt im Profil mit Columella columella erstmals eine Schneckenart der subarktischen Tundra auf. Von dem als Gräselberger Boden 1 bezeichneten Nassboden (WB3) bis in die Molluskenzonen $\mathrm{d}$ und $\mathrm{e}$ ist diese hoch kaltzeitliche Index-Art ohne Unterbrechung nachzuweisen (Abb. 6).

Die beiden alpinen Arten Orcula dolium und Neostyriaca corynodes erfahren nacheinander auffallende Bestandseinbußen. Orcula erlischt und tritt weiter oben nur noch einmal auf (WB5). Dagegen erreicht Neostyriaca im Hangenden erneut beachtliche Dominanzwerte.

Die Häufigkeit von Arten mit mehr oder weniger großem Feuchtigkeitsbedürfnis, in erster Linie Succinella oblonga, nimmt in der Vernässungszone über WB2 kontinuierlich zu. Im Löß zwischen WB2 und WB3 entfallen etwa $85 \%$ der Individuen auf diese Artengruppe. Dem entspricht ein drastischer Rückgang der Individuenzahlen bei den trockenresistenten Formen (Pupilla muscorum, Vallonia costata, Clausilia rugosa). Lediglich an der Basis von WB3 ist eine leicht gegenläufige Entwicklung festzustellen. Auch das Fehlen von Columella columella in diesem Profilabschnitt spricht für die Annahme einer trockeneren Schwankung innerhalb einer generell kalten und feuchten Phase des Mittelwürms.

\section{Zone d: Bv-Horizont WB4 (Lohner Boden)}

Das Auftreten einer vorwiegend warmzeitlichen Schneckenart (Vertigo pygmaea) neben hoch kaltzeitlichen Elementen (Columella columella, Pupilla loessica, Vertigo parcedentata) weist diesen stratigraphisch besonders markanten Horizont als interstadiale Bildung aus. Die Artenzahl (insgesamt 16) ist höher als in irgendeinem anderen Abschnitt des Profils. Auch das ist ein Hinweis auf besonders günstige Klimaverhältnisse.

\section{Zonen e und f: Umlagerungszone und Löß} mit drei Nassböden zwischen WB4 und WB5

Die Faunen aus der unteren Hälfte dieses Profilabschnittes (Abb. 6, Zone e) sind einander sehr ähnlich. Die hochglaziale Index-Art Columella columella, zuweilen in Gesellschaft von Pupilla loessica, ist überall vertreten. Auffallend häufig mit Anteilen bis zu 21,5 \% an der Gesamtzahl der Individuen sind, wie schon im oberen Bereich von WB4, die beiden Schließmundschnecken Clausilia rugosa und Neostyriaca corynodes. Insgesamt lassen die Artenspektren sowie der nach wie vor große 
Arten- und Individuenreichtum auf eine mehr oder weniger gleichförmige und weniger extreme kaltzeitliche Klimaentwicklung mit noch günstigen Lebensbedingungen für Schnecken schließen.

Gegen Ende der Phase e ist bei Pupilla muscorum eine auffallende Zunahme der durchschnittlichen Schalenmaße festzustellen, eine Erscheinung, die ursächlich mit langsamerem und verlängertem Wachstum der Tiere unter allmählich rauer werdenden Klimabedingungen in Verbindung gebracht werden kann (RoussEAU 1989). Der Übergang zu lebensfeindlichen Umweltverhältnissen spiegelt sich dann auch deutlich in den aus der oberen Hälfte dieses Profilabschnitts (Abb. 6, Zone f) überlieferten Schneckenfaunen wider. Die Artenzahl geht drastisch zurück, ebenso die Individuenzahlen. Lediglich am Beginn dieser Phase f zeichnet sich durch ein nochmaliges kräftiges Anwachsen der Individuenzahlen eine kurzzeitige, leichte Verbesserung der Lebensbedingungen ab. Arten, die zwar wenig anspruchsvoll sind, aber doch deutlich die milderen Phasen von Kaltzeiten zu bevorzugen scheinen, wie Vallonia costata oder Neostyriaca corynodes, waren nicht mehr nachzuweisen. Auch Vitrea crystallina fällt vollkommen aus.

\section{Zone g: Bereich der Bv-Horizonte WB5 und WB6}

Unter den wenigen Fossilien aus dem unteren der beiden schwachen Bv-Horizonte (WB5) befinden sich mit Abida secale und Orcula dolium zwei Schneckenarten, die als Indexarten für mildere Abschnitte der Kaltzeit angesehen werden können. Auch Vallonia costata, Vitrea crystallina und Neostyriaca corynodes, die in dem vorigen Abschnitt fehlen, sind wieder vorhanden. Die Fauna aus dem Bv-Horizont WB6 ist ganz ähnlich zusammengesetzt. Klimaempfindlichere Formen fehlen allerdings.

In der Zone g ist ein zweimaliger, kräftiger Anstieg der Individuenzahlen bemerkenswert. Die Maxima liegen direkt über WB5 sowie im obersten Abschnitt von WB6 (Abb. 3 und 6). Dazwischen sind, wie zuvor und danach, die
Individuenzahlen sehr gering. Es sind in erster Linie die drei kaltzeitlichen Häufigkeitsarten Pupilla muscorum, Succinella oblonga und Trochulus hispidus, die für die hohen Individuenzahlen in den beiden Abundanzgipfeln verantwortlich sind. In Bereich von WB5 ist Pupilla muscorum hoch dominant, was auf relativ trockene Verhältnisse schließen lässt. Im Falle von WB6 spricht die Dominanz von Trochulus hispidus und Succinella oblonga für ein erhöhtes Feuchtigkeitsangebot.

\section{Zone h: Vernässungszone, gelblicher Löß und Nassboden}

Aus diesem Bereich liegen nur einige wenige Schalenreste vor, die von 6 Arten stammen. Unter diesen befindet sich mit Columella columella eine Leitart für hochglaziale Klimaverhältnisse.

\section{Zone i: Sandstreifiger Löß und E2-Nassboden}

Auch in diesem Profilabschnitt wurden nur wenige Fossilien gefunden. Die Diversität der Fauna ist mit insgesamt 8 Arten jedoch etwas größer. Einige akzessorische Lößarten wie Vallonia costata, Clausilia rugosa, Neostyriaca corynodes und Vitrea crystallina erreichen letztmals nennenswerte Anteile. Ihre Anwesenheit lässt auf eine leichte Abschwächung eines mit Zone h einsetzenden, extremen Glazialklimas schließen.

Zone k: Löß mit E3-Nassboden, Eltviller Tuff und E4/E5-Nassbodenkomplex

In den obersten Partien des Profils hat eine Sedimentprobe selten mehr als zwei bis drei Fossilien geliefert. Eine Probe aus dem Abschnitt a des E4/E5-Nassboden-Komplexes (Abb. 3), in welcher die hygrophile Art Succinella oblonga etwas zahlreicher vorkommt (Abb. 6), bildet eine Ausnahme.

Welche Faktoren letztlich für die auffallend geringen Abundanzwerte in den meisten Abschnitten des Jungwürmlösses von Nußloch verantwortlich sind, ist schwer zu sagen. Sie 
sind selbst für hochglaziale Verhältnisse ungewöhnlich und hängen vielleicht mit den besonderen Standortsbedingungen auf dem exponierten, wachsenden Lößriedel zusammen. Hierbei ist insbesondere an extreme Austrocknung zu denken.

\subsection{Zusammenfassende Diskussion der malakozoologischen Ergebnisse}

Der Arten- und Individuenreichtum der mittelwürmzeitlichen Faunen lässt auf generell weniger extreme klimatische und ökologische Bedingungen schließen (siehe auch MoINE et al. 2005). Neben dem Lohner Boden führen noch drei weitere Horizonte thermisch anspruchsvollere Schnecken und damit wärmer getönte Faunen. Einen deutlich interstadialen Charakter hat in dem von uns bearbeiteten Profil die Fauna der Niedereschbacher Zone (Molluskenzone a). Auch aus dem Liegenden des Gräselberger Bodens vom Gräselberg bei Wiesbaden (Remy 1969: 113 und Tab.1, 6256) und aus der Niedereschbacher Zone des Lößprofils von Heilbronn-Böckingen (BIBUs \& RÄHLE 1986; RÄHLE 2004) sind solche Schneckengemeinschaften bekannt. Betrachtet man das Artenspektrum, dann ist die Fauna aus der Niedereschbacher Zone unseres Profils derjenigen Interstadial-Fauna sehr ähnlich, welche MoINE et al. (2005) aus dem so genannten „Nußlocher Boden“ beschrieben haben. Kennzeichnend sind unter anderem die Häufigkeit von Vallonia pulchella und das Fehlen von Neostyriaca corynodes, welche im Nußlocher Löß offenbar erst später auftritt.

Von den beiden Nassböden zwischen Niedereschbacher Zone und Lohner Boden, die vorläufig als "Gräselberger Boden 2 und 1“ bezeichnet werden, führt nur der untere (WB2) eine für Interstadiale charakteristische Fauna. Sie weist trotz einiger Differenzen, die auf unterschiedliche standörtliche Gegebenheiten zurückgeführt werden können, eine bemerkenswert ähnliche Zusammensetzung auf wie diejenige, welche MoINE et al. (2005) in Nußloch aus einem ebenfalls als ,Gräselberger Boden" bezeichneten Horizont beschrieben haben. Die Faunen stimmen in folgenden Merkmalen überein: (1) Abida secale tritt zum letzten Mal in nennenswerter Anzahl auf, (2) bei den Schließmundschnecken ist Neostyriaca corynodes deutlich häufiger als Clausilia rugosa parvula, nicht umgekehrt wie in allen übrigen Teilen der Lößsequenz, (3) Orcula dolium ist nirgends so häufig wie in diesem Profilabschnitt und (4) Vallonia costata erreicht höchste Dominanzwerte.

Die gleichfalls wärmer getönte Fauna, die aus dem Löß im Liegenden von WB2 vorliegt, stammt möglicherweise aus derselben wärmeren Schwankung, welche MorNe et al. (2005) in stratigraphisch ähnlicher Position bei ihren Untersuchungen in Nußloch nachgewiesen haben. Der faunistische Befund, dass hier erstmals im Profil Neostyriaca corynodes auftaucht, spricht für eine derartige Korrelation.

Die artenreichste Fauna liegt aus dem Lohner Boden (WB4) vor. Sie wird im Hangenden von ausgesprochen arten- und meistens auch sehr individuenarmen Molluskenbeständen aus dem Jungwürmlöß abgelöst. Der Faunenwandel erfolgt jedoch nicht abrupt, sondern schrittweise über zwei mildere Phasen, welche in ihrer Intensität von unten nach oben stark abnehmen (Molluskenzone e).

Von besonderem Interesse sind die beiden Abundanzgipfel, die deutliche Beziehungen zu den beiden schwachen Bv-Horizonten WB5 und WB6 aufweisen (Molluskenzone g). Sie sind in einer fast identischen stratigraphischen Position auch in einem benachbarten Nußlocher Lößprofil nachgewiesen worden (MoINE et al. 2002), und stellen eine wertvolle Hilfe bei der Parallelisierung beider Profile dar.

Die im Bereich des E2-Nassbodens (Molluskenzone i) registrierte leichte Zunahme der Artenzahl (nicht der Individuenzahl) lässt vielleicht auf einen weiteren, weniger lebensfeindlichen Zeitabschnitt schließen.

Insgesamt gesehen ergeben die paläoökologischen Untersuchungen in Nußloch ein erheblich differenzierteres Bild vom Ablauf der Klimaentwicklung im Jungwürm als früher bearbeitete, bedeutende südwestdeutsche Lößprofile wie Heilbronn-Böckingen (RÄHLE 
2004) oder vom Hainerberg bei Wiesbaden (REMY 1969), die lediglich den wenig präzisen Befund geliefert haben, dass im Bereich des E2-Nassbodens etwas anspruchsvollere Faunen auftreten. Gut vergleichen lässt sich das von uns bearbeitete Profil hingegen mit der von ANTOINE et al. (2001) untersuchten Nußlocher Lößsequenz. Die von dort vorliegenden malakozoologischen Befunde (MorNe et al. 2002) stimmen mit unseren Ergebnissen weitgehend überein.

\section{Befunde der Lumineszenz-Datierungen in Nußloch}

\subsection{Lumineszenz-Datierungen}

Mit Hilfe der Lumineszenz-Datierungsverfahren kann das Sedimentationsalter, d.h. die Zeit, die seit der letzten Nullstellung durch Sonnenlicht-Exposition vergangen ist, von Lockersedimenten bestimmt werden. Zusammenfassende Darstellungen über den Stand der Forschung finden sich in Duller (2004) sowie Lian \& RoBerts (2006). Die physikalischen Grundlagen werden ausführlich in den Monographien von Aitken (1998) und Boetter-Jensen et al. (2003) dargestellt. In dieser Arbeit wird die Infrarot Optisch Stimulierte Lumineszenz (IRSL) angewendet (FRECHEN et al. 2007). IRSL-Datierungen wurden erfolgreich an äolischen Sedimenten wie Lössen, Dünen und Flugsanden angewendet (FRECHEN et al. 1995).

Die IRSL-Datierungsobergrenze hängt u.a. von der Dosisleistung, d.h. von der natürlichen radioaktiven Strahlung im Sediment, und der Mineralart (Quarz oder Feldspat) ab. Für viele mitteleuropäische Löß-/Paläobodenabfolgen liegen stratigraphisch konsistente Alter bis zu etwa 150.000 Jahren vor heute vor (FRECHEN et al. 2003; Novothny et al. 2002). Allerdings weisen Lumineszenz-Alter von etwa 100.000 Jahren bereits signifikante Altersunterbestimmungen von etwa 20-30 \% auf (FRECHEN 1992).

Die Aufbereitung von Lumineszenz-Proben findet im Rotlichtlabor statt. Es wurde polymineralisches Feinkornmaterial der Fraktion 4-11 $\mu \mathrm{m}$ aufbereitet. Karbonate und organische
Beimengungen wurden mit Salzsäure und Wasserstoffperoxid gelöst. Die Korngrößenfraktion 4-11 $\mu \mathrm{m}$ wurde über mehrere Trenngänge mittels Zentrifuge angereichert. Das aufbereitete Probenmaterial wird mit Aceton in Suspension gebracht und auf 50 Aliquots mittels Pipette gleichmäßig aufgetragen (FRECHEN et al. 1996). Eine kalibrierte ${ }^{90} \mathrm{Sr} /{ }^{90} \mathrm{Y}$-Betaquelle wurde zum künstlichen Bestrahlen verwendet, um herauszufinden, wie das Probenmaterial auf sukzessive größer werdende Bestrahlungsdosen reagiert. Jeweils fünf Aliquots wurden in sukzessive aufsteigenden Dosisschritten zwischen 45 Gray (Gy) und 750 Gy bestrahlt, mit dem Ziel die Äquivalentdosis zu bestimmen. Die Äquivalentdosis ist die Dosis, die die Minerale seit der letzten Sonnenlicht-Exposition bzw. der Ablagerung absorbiert haben. Die Aliquots wurden nach der Bestrahlung für 4-6 Wochen bei Raumtemperatur gelagert, um instabile Signale wie beispielsweise ,anomalous fading“ (WintLe 1973) abklingen zu lassen. Alle Aliquots wurden vor der Messung eine Minute lang bei $230^{\circ} \mathrm{C}$ vorgeheizt, um thermisch instabile Signale zu eliminieren. Eine Schott BG39/ Corning 7-59 Filterkombination wurde bei den Messungen Photomultiplier und Aliquots zwischengeschaltet, um ausschließlich die stabilere blaue Emission zu messen. Die Äquivalentdosis wurde mittels „Multiple-Aliquot-Additive Dose (MAAD)"-Protokoll bestimmt (WINTLE 1997). Die IRSL-Zerfallskurven wurden über das Integral 1-5 s integriert. Mit dem Programm Analyst 6.0 (G.A.T. Duller, Aberystwyth/Risö) wurde eine exponentielle Wachstumskurve über die Messwerte extrapoliert und mit der natürlichen Lumineszenz verglichen, um so die Äquivalentdosis zu bestimmen.

Die Alpha-Effektivität wurde für alle Proben mit $0,08 \pm 0,02$ angenommen (vgl. LANG et al. 2003). Die Konzentration relevanter Radioelemente wurde gammaspektrometrisch bestimmt (FreChen et al. 2007). Die Berechnung der Dosisleistung erfolgte über die gammaspektrometrisch detektierte Konzentration der radioaktiven Elemente sowie ihrer Tochterprodukte: Kalium $\left({ }^{40} \mathrm{~K}\right.$ bei 1560.8 $\mathrm{keV})$, Uran $\left({ }^{214} \mathrm{~Pb}\right.$ bei $295.2 \mathrm{keV}$ und 351.9 
$\mathrm{keV},{ }^{214} \mathrm{Bi}$ bei $609.3 \mathrm{keV}$ und $1129.3 \mathrm{keV}$ ) und Thorium $\left({ }^{228} \mathrm{Ac}\right.$ bei $338.3 \mathrm{keV}$ und $911.1 \mathrm{keV}$, ${ }^{208} \mathrm{Tl}$ bei $538.2 \mathrm{keV}$ und $2614.5 \mathrm{keV},{ }^{212} \mathrm{Bi}$ bei $727.3 \mathrm{keV}$ ). Ein radioaktives Gleichgewicht wurde für die Zerfallsketten vorausgesetzt. Die kosmische Dosisleistung ist abhängig von den Koordinaten (Längen- und Breitengrad) der Probenlokalität, der Höhe über Normal Null und der überlagernden Sedimentmächtigkeit (Aitken 1998; Prescott \& Hutton 1994). Der natürliche Wassergehalt wurde für alle Proben mit $20 \pm 5 \%$ angenommen.

\subsection{Resultate der Lumineszenzdatierungen}

Aus dem Aufschluss Nußloch wurden 27 Proben für Lumineszenz-Datierungen entnommen. Die dosimetrischen Ergebnisse sowie die Altersergebnisse finden sich in Tabelle 1 und Abbildung 3. Der Urangehalt schwankt zwischen 1,7 und 2,8 ppm, der Thoriumgehalt zwischen 6,6 und 10,2 ppm und der Kaliumgehalt zwischen 0,86 und $1,41 \%$. Die daraus errechnete Effektiv-Dosisleistung liegt zwischen 2,38 und 3,21 Gy/ka. Diese dosimetrischen Werte sind typisch für mitteleuropäischen Lösse (FRECHEN

Tab. 1: Analytische Ergebnisse und IRSL-Altersbestimmungen der Proben aus Nußloch. Die Probenfeuchte wurde auf $20 \pm 5 \%$ und die Alpha-Effektivität auf $0.08 \pm 0.02$ für alle Proben festgelegt. De: Äquivalentdosis in Gray (Gy); ka: 1000 Jahre.

Tab. 1: Analytical results of IRSL age determination for the samples from the Nussloch section. The moisture was estimated to $20 \pm 5 \%$, the alpha efficiency to $0.08 \pm 0.02$ for all samples. De: equivalent dose in Gray (Gy); ka: 1000 years.

\begin{tabular}{|c|c|c|c|c|c|c|c|c|c|}
\hline Sample & $\begin{array}{l}\text { Lab.- } \\
\text { Ident. } \\
\text { LUM }\end{array}$ & $\begin{array}{l}\text { Depth } \\
\text { [m] }\end{array}$ & $\begin{array}{l}\mathrm{U} \\
{[\mathrm{ppm}]}\end{array}$ & $\begin{array}{l}\text { Th } \\
{[\mathrm{ppm}]}\end{array}$ & $\begin{array}{l}\mathrm{K} \\
{[\%]}\end{array}$ & $\begin{array}{l}\text { Cosmic } \\
{[\mu \mathrm{Gy} / \mathrm{a}]}\end{array}$ & $\begin{array}{l}\text { Dose } \\
\text { Rate } \\
{[\text { Gy/ka] }}\end{array}$ & $\begin{array}{c}\text { ED } \\
{[\mathrm{Gy}]}\end{array}$ & $\begin{array}{l}\text { IRSL } \\
\text { age } \\
\text { [ka] }\end{array}$ \\
\hline Bil & 145 & 17.00 & $80 \pm 0.14$ & $9.50 \pm 0.48$ & $1.32 \pm 0.07$ & $76 \pm 4$ & $3.18 \pm 0.33$ & 239. & $75.3 \pm 7$ \\
\hline $\mathrm{Bi} 2$ & 46 & 18.10 & $49 \pm 0.12$ & $10.20 \pm 0.51$ & $1.41 \pm 0.07$ & $72 \pm 4$ & $3.21 \pm 0.33$ & $157.9 \pm 4.2$ & $49.2 \pm 5.6$ \\
\hline $\mathrm{Bi} 3$ & 47 & .70 & $1.71 \pm 0.09$ & $6.56 \pm 0.33$ & $1.20 \pm 0.06$ & $81 \pm$ & .23 & 135.5 & $57.0 \pm 5.7$ \\
\hline $\mathrm{Bi} 4$ & 48 & 15.30 & $2.12 \pm 0.11$ & $7.99 \pm 0$ & $1.06 \pm 0.05$ & $83=$ & 2.57 & 104.0 & $40.5 \pm 4.3$ \\
\hline $\mathrm{Bi5}$ & 49 & 15.00 & $2.46 \pm 0.12$ & $9.77 \pm($ & $1.21 \pm 0.06$ & $84 \pm 4$ & & 104. & $34.9 \pm 3.8$ \\
\hline Bi6 & 0 & 50 & $2.27=$ & 8.45 & 0.95 & 87 & & 94. & $36.6 \pm 3.9$ \\
\hline Bi7 & 51 & 0 & 2.23 & 7.99 & 5 & & & & $34.0 \pm 3.7$ \\
\hline $\mathrm{Bi} 8$ & & & 2 & 8.2 & & & & & $27.4 \pm 2.9$ \\
\hline Bi9 & 3 & 1 & 2.36 & & 0.9 & 93 & & & $33.5 \pm 3.8$ \\
\hline $\mathrm{Bi10}$ & 54 & & & & 0.86 & & & & $31.7 \pm 3.6$ \\
\hline Bil1 & - & & & & 1.1 & & & & $26.7 \pm 3.4$ \\
\hline Bil & 56 & & & & & & & & $26.0 \pm 2.9$ \\
\hline $\mathrm{Bi13}$ & 57 & & & & & & & & $25.9 \pm 3.7$ \\
\hline Bil & 58 & & & & & & & & $32.0 \pm 3.7$ \\
\hline Bil & 59 & & & & & & & & $25.1 \pm 2.8$ \\
\hline Bil & 60 & & & & & & & & $27.7 \pm 3.0$ \\
\hline Bil & 61 & & & & & & & & $25.2 \pm 2.7$ \\
\hline Bil & 62 & & & & & & & & $23.4 \pm 2.7$ \\
\hline $\mathrm{Bi}$ & 78 & & & & & & & & $19.7 \pm 2.3$ \\
\hline $\mathrm{Bi2}$ & 164 & & & & & & & 53. & $19.4 \pm 2.2$ \\
\hline $\mathrm{Bi} 2$ & 165 & & & & & 125 & & & $24.4 \pm 2.9$ \\
\hline Bi22 & 166 & & & & & $126 \pm 6$ & & 48. & $19.1 \pm 2.4$ \\
\hline $\mathrm{Bi} 23$ & 167 & 7.4 & & & & $131 \pm 7$ & & $57.5 \pm 2.1$ & $21.0 \pm 2.3$ \\
\hline Bi24 & 168 & 7.00 & & & & & & & $20.7 \pm 2.2$ \\
\hline Bi25 & 169 & & & & & & & & $18.6 \pm 2.0$ \\
\hline $\mathrm{Bi26}$ & 170 & & & & & & & & $23.2 \pm 2.6$ \\
\hline Bi27 & 171 & & $2.27 \pm 0.11$ & $8.01 \pm 0.40$ & $1.13 \pm 0.06$ & $205 \pm 10$ & $2.80 \pm 0.27$ & $52.3 \pm 4.2$ & $18.7 \pm 2.4$ \\
\hline
\end{tabular}


et al. 2003); sie stimmen ebenfalls ausgezeichnet mit den Ergebnissen von LANG et al. (2003) aus einem früher bearbeiteten Profil im gleichen Aufschluss in Nußloch überein. Die Dosisleistung ist für Bodenmaterial aus dem Bt-Rest und dem umgelagerten Bodensediment darüber am größten. Die niedrigste Dosisleistung wurde für sandige Lösse analysiert.

Die Äquivalentdosis reicht von 48,7 $\pm 3,4$ Gy bis zu 239,6 $\pm 2,8$ Gy. Der überwiegende Teil der Aufbaukurven liegt im linearen Auswertebereich. Aufgrund der exzellenten Reproduzierbarkeit der Messungen wurden von einer Normalisierung der Einzelmessungen abgesehen. Die IRSL-Alter lassen sich in vier Altersgruppen unterteilen (vgl. Tab. 1).

Die stratigraphisch älteste Probe wurde aus dem Ah-Horizont der Mosbacher Humuszone (Boden WB1 im Abschnitt B) genommen. Sie ergibt ein ISRL-Alter von 75,3 \pm 7,8 ka (Probe 1). Eine Korrelation mit der jüngsten Mosbacher Humuszone (s. auch Frechen \& Preusser 1996) ist aus chronologischen Gründen sehr wahrscheinlich.

Der nächste Altersbereich umfasst vorwiegend das Mittelwürm zwischen 12,50 und $18,10 \mathrm{~m}$ unterhalb Geländeoberkante (GOK, Tiefenangaben in diesem Kapitel beziehen sich auf die Angaben in Tab. 1). Es handelt sich um die Proben 2 - 11 im Abschnitt C. Die IRSL-Alter reichen von $57,0 \pm 5,7 \mathrm{ka}$ bis $26,7 \pm 3,4 \mathrm{ka}$. Der kräftige braune Boden (Boden WB4), der diesen Abschnitt abschließt, wird zeitlich mit dem Lohner Boden korreliert. Diese Schlussfolgerung basiert auf der Tatsache, dass sämtliche Proben unterhalb des Bodens ein junges mittelwürmzeitliches Alter besitzen. Der Boden selbst hat jedoch mit 26,7 $\pm 3,4$ ka (Probe 11) ein geringfügig zu junges Alter, stimmt jedoch innerhalb der 1-sigma Standardabweichung mit dem erwarteten Alter überein. Allerdings kann dieser Boden nach den Geländebefunden geringfügig verlagert und damit etwas zu jung sein. Eine ${ }^{14} \mathrm{C}$-Datierung an Schnecken mit einem Alter von $31,660 \pm 310$ BP (KIA 12348) bestätigt eine Zuordnung zum Lohner Boden. Mit den Proben 12 - 17 sind sechs Proben aus der Sedimentabfolge zwischen 9,00 und
12,00 m unterhalb GOK entnommen worden (Abschnitt D). Die IRSL-Alter reichen von $32,0 \pm 3,7 \mathrm{ka}$ bis $23,4 \pm 2,7 \mathrm{ka}$ und sind somit numerisch in das Jung- bzw. Oberwürm zu stellen. Die Probe B14 (32,0 $\pm 3,7 \mathrm{ka})$ mit höherem IRSL-Alter liegt in einem Nassbodenbereich mit solifluidaler Umlagerung. Es ist deshalb zu vermuten, dass das Sediment vor der Ablagerung nicht vollkommen gebleicht wurde.

Für die beiden abschließenden schwachen Böden (WB5, WB6) und das dazwischen liegende Sediment wurden Alter zwischen 25,2 \pm 2,7 ka und 19,4 $\pm 2,2$ ka bestimmt. Nach den IRSLAltern können deshalb diese beiden Horizonte nicht mit dem Lohner und Böckinger Boden parallelisiert werden, da die Alter zu jung sind. Dies lässt sich auch durch die ${ }^{14} \mathrm{C}$-Alter bestätigen. So hat die Vernässungszone des unteren schwachen Bodens (WB5) ein ${ }^{14} \mathrm{C}$ Alter von $24.790 \pm 160 \mathrm{BP}$ (KIA 12347) und die des oberen schwachen Bodens (WB6) ein ${ }^{14} \mathrm{C}$-Alter von $23.870 \pm 170 \mathrm{BP}$ (KIA 12346) an Schnecken ergeben.

Der oberste Bereich der Abfolge zwischen 0,70 und 9,00 m unterhalb GOK erbrachte IRSLAlter von $18,6 \pm 2,0 \mathrm{ka}$ und $24,2 \pm 2,9 \mathrm{ka}$. Dieser Teil des Profils korreliert chronologisch eindeutig mit dem Oberwürm (Jungwürm). Durch den Nachweis der mafischen Asche des Eltviller Tuffs in ca. 4,50 m unter GOK war bereits schon vor den numerischen Datierungen klar, dass es sich bei den darüber liegenden Nassböden zweifelsfrei um den E4/E5-Nassbodenbereich handelt. Dieser Abschnitt wurde deshalb nicht beprobt. Die IRSL-Daten zeigen insgesamt, dass in dem untersuchten Lößrücken von Nußloch innerhalb des Oberwürms (Jungwürms) in gut 12.000 Jahren bis zu $12 \mathrm{~m}$ Löß bei einer hohen Sedimentationsrate abgelagert wurde, weshalb es zu einer sehr starken Differenzierung der Nassböden, insbesondere im E2- und E4/E5-Bereich, gegenüber den normal gegliederten Standardprofilen gekommen ist.

Die Lumineszenz-Datierungen stimmen ausgezeichnet mit einer weiteren Datierungsstudie aus Nußloch überein (LANG et al. 2003). Die IRSL und ${ }^{14} \mathrm{C}$-Alter aus dieser Arbeit kann man 
ebenfalls in vier Altersabschnitte unterteilen:

1. die älteste Probe ergab ein IRSL-Alter von $122,0 \pm 17,8$ ka und korreliert vermutlich mit dem Riß,

2. zwischen 15,45 und $18,55 \mathrm{~m}$ unterhalb GOK wurden IRSL-Alter von 55,7 $\pm 12,6 \mathrm{ka}$ bis 61,3 $\pm 9,9$ ka bestimmt,

3. zwischen 11,00 und $12,70 \mathrm{~m}$ unterhalb GOK liegen die IRSL-Alter zwischen 29,5 $\pm 3,7 \mathrm{ka}$ und $34,0 \pm 3,2 \mathrm{ka}$, und

4. zwischen $1,20 \mathrm{~m}$ und $9,65 \mathrm{~m}$ unterhalb GOK wurden Alterswerte von 18,2 $\pm 3,7 \mathrm{ka}$ und 26,0 $\pm 4,0$ ka bestimmt.

Für das Profil Nußloch liegt somit ein hervorragender chronologischer Rahmen vor, der es ermöglicht, dieses hoch auflösende würmzeitliche Sedimentarchiv mit weiteren zeitgleichen Sequenzen regional und überregional zu korrelieren.

\section{Zusammenfassung}

In Nußloch südlich von Heidelberg wurde im Aufschluss der Heidelberger Zement AG beim Abraumabbau ein von Dellen flankierter Lößriedel vollkommen durchschnitten. Die aufgeschlossenen Horizonte zeichnen die Oberfläche des Riedels nach, so dass von einer stetigen Aufschüttung ausgegangen werden kann. Dadurch kam es zu einer sehr differenzierten Profilabfolge, die in mehreren Abschnitten weit über die klassische Würmlößgliederung hinausgeht (vgl. Schönhals et al. 1964; SemMEL 1968).

An anderer Stelle des Aufschlusses wurden von Antolne et al. (2001) mehrere Profile aufgenommen, die jedoch z.T. nicht die Reichhaltigkeit des Bodeninventars in Superposition wie im neu bearbeiteten Aufschluss erreichen. Neben der Gesamtaufnahme des zentralen Riedelbereiches wurde ein Standardprofil im Würmlöß mit dem liegenden Eemboden von insgesamt ca. $18 \mathrm{~m}$ erarbeitet und mit zahlreichen Methoden untersucht. Den chronologischen Rahmen geben durchgeführte IRSL- und ${ }^{14} \mathrm{C}$-Datierungen. Bodentypologisch konnte ein mehrgliedriger Bt-Horizont, eine Humuszone, zahlreiche einfache Nassböden sowie mehrere
Nassböden mit unterschiedlich mächtigen Verbraunungshorizonten nachgewiesen werden. Da keine der Verbraunungszonen entkalkt ist, handelt es sich um initiale $\mathrm{Bv}$-Horizonte $(\mathrm{Bv}-$ $\mathrm{Cv}$ ) vom Typ der arktischen Braunerden.

Zwischen dem letztinterglazialen 1.fBt-Horizont und dem Eltviller Tuff wurden in der Würmsequenz, wenn man von der AltwürmHumuszone (WB1) absieht, noch fünf mehr oder weniger deutliche Verbraunungszonen mit überlagernden Nassböden (WB2 - WB6) ausgegliedert.

Der kräftigste $\mathrm{Bv}$-Horzont ist der Boden WB4 in ca. $12 \mathrm{~m}$ Tiefe. Aufgrund seiner Eigenschaften, seines IRSL- und ${ }^{14} \mathrm{C}$-Alters sowie seiner herausstechenden, artenreichen, interstadialen Molluskenfauna wird er als Äquivalent des Lohner Bodens angesehen. Im darunter liegenden Mittelwürmlöß sind zwei Bodenbildungen vorhanden (WB2, WB3), die mit dem Gräselberger Boden parallelisiert werden und von denen auf jeden Fall die untere aufgrund der Molluskenführung auch als interstadial einzustufen sind. Bei der darunter folgenden Altwürm-Humuszone (WB1) handelt es sich nach den IRSL-Datierungen mit großer Wahrscheinlichkeit um die jüngste der drei Mosbacher Humuszonen. Im Alt- und Mittelwürmlöß muss deshalb insgesamt mit mindestens sechs Interstadialen einschließlich des Lohner Bodens gerechnet werden. Bei der Reichhaltigkeit des Profils tritt allerdings die Frage auf, warum der Böckinger Boden fehlt, da eine Deutung der beiden Gräselberger Böden als Böckinger Boden typologisch nicht möglich ist. Vermutlich liegt deshalb im Mittelwürmlöß eine nicht zu erkennende Diskordanz vor.

Der Jungwürmlöß erreicht mit $12 \mathrm{~m}$ eine große Mächtigkeit. Vor allem der Abschnitt zwischen dem Lohner Boden und dem Eltviller Tuff umfasst zahlreiche im Löß eingeschaltete Nassböden, von denen zwei (WB5 und WB6) eine deutliche Verbraunung aufweisen. Es wurde deshalb am Anfang unserer Untersuchungen diskutiert, ob die beiden Böden nicht dem Lohner und dem Böckinger Boden entsprechen. Die absoluten Datierungen $\left(\mathrm{IRSL},{ }^{14} \mathrm{C}\right)$ und die relativ schwache Ausbildung lassen eine sol- 
che Deutung nicht zu. Der gesamte Abschnitt zwischen dem Lohner Boden und dem Eltviller Tuff muss dem E1- bis E3-Nassbodenbereich und dabei vor allem dem auch an anderen Stellen mehrgliedrigen E2-Abschnitt entsprechen. Allerdings ist bislang in keinem anderen Lößprofil in Mitteleuropa eine so starke Aufgliederung beobachtet worden.

Auch im Jungwümlöß sind einige, allerdings schwächer ausgebildete Schwankungen zu klimagünstigeren Abschnitten festzustellen. Am deutlichsten sind sie in den beiden schwachen Verbraunungszonen WB5 und WB6 ausgebildet. Im oberen Bereich dieser beiden Horizonte ist bei den Mollusken jeweils eine starke $\mathrm{Zu}$ nahme der Individuenzahlen zu verzeichnen. Diese beiden Abundanzgipfel wurden auch in einem benachbarten Profil in Nußloch gefunden und sind deshalb neben dem Eltviller Tuff eine wertvolle Hilfe zur Parallelisierung beider Profile (Moine et al. 2002). Nach den Datierungen $\left(\right.$ IRSL, ${ }^{14} \mathrm{C}$ ) sind die beiden Böden zwischen $25 \mathrm{ka}$ und $20 \mathrm{ka}$ BP einzustufen. Auch ANTOINE et al. (2001) haben für diesen Bereich ähnliche Alter festgestellt. Vielleicht ist auch die von Sommé et al. (1986) beschriebene, interstadiale Molluskenfauna von Achenheim (Elsaß) hierher zu stellen, die zwischen dem „loess récent inférieur“ und dem „loess récent supérieur" liegt und bei der TL-Datierungen auf ein Alter von 20 bis $25 \mathrm{ka}$ BP hinweisen (ZöLLER 1995).

Bei den kalten Phasen vor und nach dieser(n) Wärmeschwankung(en) dürfte es sich um die kältesten Abschnitte der Würmeiszeit gehandelt haben, für welche nach GuIot et al. (1993) die Zeitabschnitte zwischen 29 und 26 ka BP sowie 21 und 20 ka BP (Heinrich Events 3 und 2) in Frage kommen.

Relativ schwach sind die Hinweise auf das Vorliegen einer weiteren wärmeren Schwankung im Liegenden des E3-Nassbodens, wo die Diversität der Molluskenfauna leicht zunimmt und die Suszeptibilitätswerte leicht erhöht sind.

Über dem Eltviller Tuff ist der Bereich des E4und E5-Nassbodens mehrgliedrig ausgebildet, weshalb von einem E4/E5-Nassbodenkomplex gesprochen wurde. Es lassen sich mindestens fünf Vernässungshorizonte (vgl. Abb. 6, Abschnitt F, a-e) ausgliedern, wobei im unteren Bereich drei schwache Verbraunungszonen im Löß angedeutet sind. ZÖLLER \& LÖSCHER (1999) haben deshalb in diesem Abschnitt mit dem Maisbacher Boden einen weiteren schwach verbraunten Nassboden gegenüber der klassischen Lößgliederung ausgeschieden.

Bemerkenswert ist beim unteren Nassbodenbereich im E4/E5-Komplex, der dem E4Nassboden zuzuordnen ist, das Auftreten von Phytolithen von Wiesen- und Waldgräsern, Moosen und zweikeimblättrigen Pflanzen, die auf einen lichten Laubwald hinweisen. Bei den Mollusken gibt es keinerlei Anzeichen für eine solche Entwicklung. Ansonsten stimmen die biomorphologischen und malakologischen Untersuchungen weitgehend überein, die für alle Abschnitte des Mittel- und Jungwürmlösses eine offene Landschaft belegen.

Die Schlussfolgerungen, die sich bezüglich der klimatischen Entwicklung aus der Paläobodensequenz und der Veränderung der Molluskenfauna ziehen lassen, stimmen erstaunlich gut mit der Kurve der magnetischen Suszeptibilität überein. Sämtliche als interstadial erkannte Abschnitte spiegeln sich in einer mehr oder minder starken Erhöhung der Suszeptibilität wider. Eine Ausnahme bildet lediglich der Bereich des Gräselberger Bodens 2 (WB3, Molluskenzone c; Abb. 3 und 6), wo trotz erhöhter Suszeptibilitätswerte faunistisch eine deutliche Abnahme sowohl der Diversität als auch der Abundanz festzustellen war.

Die Naßboden/Lößsequenzen im Jungwürmlöß, welche möglicherweise kurzfristige Klimaschwankungen zwischen kalttrockenen und kaltfeuchten Abschnitten widerspiegeln, stimmen im Übrigen weitgehend mit den DansgaardOeschger-Events zwischen ca. 31 und 19 ka BP in den GRIP-Bohrungen im grönländischen Eis überein (vgl. auch RousseAu et al. 2002).

Ein pedologischer Vergleich mit den von ANTOINE et al. (2001), ZÖLLER (1995) und ZÖLLER \& LÖSCHER (1999) beschriebenen Profilen in Nußloch zeigt auf den ersten Blick Übereinstimmungen mit unseren Aufnahmen, im Detail ergeben sich jedoch Parallelisierungs- 
schwierigkeiten. Dies gilt vor allem für den Bereich des Mittelwürmlösses. Antorne et al. (2001) weisen in ihrem Profil P1 zwar einen Gräselberger Boden aus, doch kann es sein, dass der Bereich der Gräselberger Böden in diesem Profil tatsächlich tiefer liegt. Es ist dabei nicht auszuschließen, dass der von ZöLLER (1995) neu eingeführte Nußlocher Boden einem der beiden von uns ausgewiesenen Gräselberger Böden entspricht. Weiterhin tritt im unteren Bereich des Profils P1 bei AnToINe et al. (2001) mit dem Horizont 9 ein Bv-Horizont vom Typ der arktischen Braunerde auf, der bei der Gesamtinterpretation zu wenig berücksichtigt wurde. Hinweise für das in Rinnen eingeschnittene und durch Fossilfunde ausgezeichnete Nußlocher Interstadial konnten in dem neu untersuchten Profil nicht gefunden werden. Mit dem im Hangenden auftretenden Lohner Boden beschreiben ANTOINE et al. (2001) drei dicht hintereinander folgenden Interstadiale („Gräselberger Boden“, Nußloch-Interstadial, Lohner Boden) im oberen Mittelwürmlöß. Es dürfte sich hierbei um den Abschnitt des Lohner und Böckinger Bodens (BIBus 1989) in den bisher beschriebenen Profilen von BadenWürttemberg handeln. Am Schwalbenberg bei Remagen ist dieser Abschnitt wie bei ANTOINE et al. (2001) dreigeteilt. Hier handelt es sich um maximal drei kräftige $\mathrm{Bv}$-Horizonte (vgl. BiBus 1995; SCHIRMER 2000).

In dem von uns bearbeiteten Profil zeigt der Lohner Boden mit der darüber liegenden Jungwürmsequenz aus Nassböden und zwischengelagertem Löß eine weitgehende Übereinstimmung mit dem Profil P2 von Antorne et al. (2001). Nur der E4/E5-Komplex ist in dem neu bearbeiteten Profil wesentlich mächtiger und besser gegliedert (s.o.).

Abschließend sei noch erwähnt, dass es im Aufschluss von Nußloch auch JungwürmlößAbfolgen gibt, die von der Bodenabfolge und der Lößmächtigkeit her vollkommen den bisher bekannten Standardprofilen entsprechen. Der Jungwürmlöß ist deshalb nur in gewissen geomorphologischen Positionen (Riedeln, Dünen) so extrem mächtig abgelagert worden.

\section{Danksagungen}

Wir danken Herrn Prof. Dr. J. KIND für Hinweise und Anregungen zur Bearbeitung des Profils, Herrn Prof. Dr. L. ZöLler für die Zustimmung zur Untersuchung, Herrn Dr. M. Löscher für Profilpräparierungen und Herrn Betriebsleiter RothENACHER für technische Unterstützungen. Weiterhin danken wir Frau Prof. Dr. A. GolyEVA (Geographisches Institut Moskau) für paläobotanische Untersuchungen und Herrn Prof. Dr. E. Appel (Universität Tübingen) für die Benutzung des Geophysikalischen Labors. M. FRECHEN bedankt sich bei der Deutschen Forschungsgemeinschaft für die finanzielle Förderung im Rahmen des Projektes ,Sediment budget of Pliocene and Quaternary sediments in the Upper Rhine area“" (HI 643/2-3).

\section{Literatur}

AitKen, M.J. (1998): Introduction to Optical Dating. - 267 S.; Oxford (Oxford University Press).

Antoine, P., Rousseau, D.-D., Zöller, L., Lang, A., Munaut, A.-V., Hatté, C. \& Fontugne, M. (2001): High-resolution record of the last Interglacial-glacial cycle in the Nussloch loess-palaeosol sequences, Upper Rhine Area, Germany. - Quaternary International, 76/77: 221-229; Oxford.

BENTE, B. (1987): Geochemische, sedimentologische und stratigraphische Untersuchungen an LößProfilen des westlichen Kraichgaus. - Dipl.-Arb. Geol.-Paläontol. Inst. Univ. Heidelberg: 107+26 S.; Heidelberg.

Bente, B. \& Löscher, M. (1987): Sedimentologische, pedologische und stratigraphische Untersuchungen an Lössen südlich Heidelberg. - Göttinger geogr. Abh., 84: 9-17; Göttingen.

BiBus, E. (1973): Ausbildung und Lagerungsverhältnisse quartärer Tuffvorkommen in der Wetterau. - Notizbl. hess. L.-Amt Bodenforsch., 101: 346361; Wiesbaden.

BiBUS, E. (1974): Abtragungs- und Bodenbildungsphasen im Rißlöß. - Eiszeitalter u. Gegenwart, 25: 166-182; Öhringen.

Bibus, E. (1980): Zur Relief-, Boden- und Sedimententwicklung am unteren Mittelrhein. - Frankfurter geowiss. Arb., Serie D, 1: 296 S.; Frankfurt a.M.

Bibus, E. (1989): 8. Tagung das AK „Paläoböden“ 
der dt. bodenkdl. Ges. vom 25.05. - 27.05. 1989 in Heilbronn. Programm u. Exkursionsführer. Mit Beitr. von W. RäHLE und L. ZöLlER. - 31 S.; Tübingen.

Bibus, E. (1995): Geomorphologische Position der Fundstelle und Gliederung der Lösse am Schwalbenberg bei Remagen. - In: App, V. et al. (Hrsg.): Die altsteinzeitliche Fundstelle am Schwalbenberg bei Remagen. - Ber. Archäologie Mittelrhein u. Mosel, Trierer Z., Bh. 20: 15-20; Trier.

BiBus, E. (2002): Zum Quartär im mittleren Neckarraum - Reliefentwicklung, Löß/Paläobodensequenzen, Paläoklima. - Tübinger geowiss. Arb., Serie D, 8: 1-276.; Tübingen.

Bibus, E. \& RÄHLE, W. (1986): Geomorphologische Lage, Deckschichten und Molluskenführung letztinterglazialer Hochflutlehme im Neckartal (Baden-Württemberg). - Eiszeitalter u. Gegenwart, 36: 86-109; Hannover.

Bibus, E., Bludau, W., Bross, C. \& RÄhle, W. (1996): Der Altwürm- und Rißabschnitt im Profil MainzWeisenau (Heidelberger Zement AG) und die Eigenschaften der Mosbacher Humuszonen. - Frankfurter geowiss. Arb., Serie D, 20: 21-52; Frankfurt a. M.

Bibus, E. \& Strahl, M. (2000): Zur Gliederung und Altersstellung der bayerischen Hochterrassen nördlich des Donauriedes. - Z. Geomorph., N.F., 44 (2): 211-232; Berlin, Stuttgart.

Boetter-Jensen, L., McKeever, S.W.S. \& Wintle, A.G. (2003): Optically stimulated luminescence dosimetry. - 404 S.; Amsterdam (Elsevier).

Duller, G.A.T. (2004): Luminescence dating of Quaternary sediments: recent advances. - Journal of Quaternary Sciences, 19: 183-192; London.

Fassbinder, J.W.E., Stanjek, H. \& Vali, H. (1990): Occurence of magnetic bacteria in soil. - Nature, 343: 161-163; London.

Florindo, F., Zhu, R. \& Guo, B. (1999): Low field susceptibility and palaeorainfall estimates. New data along a N-S transect of the Chinese loess plateau. - Physics Chemistry Earth, A 24 (9): 817-821; Amsterdam, Oxford.

FreChen, M. (1992): Systematic thermoluminescence dating of two loess profiles from the Middle Rhine Area (F.R.G.). - Quaternary Science Reviews, 11: 93-101; Oxford.

Frechen, M., Boenigk, W. \& Weidenfeller, M. (1995): Chronostratigraphie des „Eiszeitlichen Lößprofils“" in Koblenz-Metternich. - Mainzer geowiss. Mitt., 24: 155-180; Mainz.
Frechen, M., Oches, E.A. \& Kohfeld, K.E. (2003): Loess in Europe - mass accumulation rates during the Last Glacial Period. - Quaternary Science Reviews, 22: 1835-1857; Oxford.

Frechen, M. \& Preusser, F. (1996): Kombinierte Lumineszenz-Datierungen am Beispiel des Lößprofils Mainz-Weisenau. - Frankfurter geowiss. Arb., Serie D, 20: 53-66; Frankfurt a. M.

Frechen, M., Schweitzer, U. \& Zander, A. (1996): Improvements in sample preparation for the fine grain technique. - Ancient TL, 14/2: 15-17; Clermont-Ferrand.

Frechen, M., Terhorst, B. \& Rähle, W. (2007): The Upper Pleistocene loess/palaeosol sequence from Schatthausen in North Baden-Württemberg. - Eiszeitalter u. Gegenwart, 56/3: 212-226; Hannover.

Guiot, J., de Beaulieu, J.-L., Chaddadi, R., David, F., Ponel, P. \& Reille, M. (1993): The climate in western Europe during the last glacial/ interglacial cycle derived from pollen and insect remains. - Palaeogeography, Palaeoclimatology, Palaeoecology, 103: 73-79; Amsterdam.

Hatté, C., Antoine, P., Fontugne, M., Rousseau, D.D., Tisnerat-Laborde, N. \& Zöller, L. (1999): New chronology and organic matter $\partial^{13} \mathrm{C}$ paleoclimatic significance of Nussloch loess sequence (Rhine Valley, Germany). - Quaternary International, 62: 85-91; Oxford.

Hatté, C., Antoine, P., Fontugne, M., Lang, A., Rousseau, D.-D. \& Zöller, L. (2001): $\partial^{13} \mathrm{C}$ of loess organic matter as a potential proxy for a paleoprecipitation reconstruction. - Quaternary Research, 55: 33-38; Washington.

Heller, F., Shen, C.D., Beer, J., Liux, M., Liu, T.S., Bronger, A., Suter, M. \& Bonani, G. (1993): Quantitative estimates of pedogenic ferromagnetic mineral formation in Chinese loess and palaeoclimatic implications. - Earth and Planetary Science Letters, 114: 385-390; Amsterdam.

Heller, F. \& Evans, M.E. (1995): Loess magnetism. - Reviews of Geophysics, 33: 211-240; Washington.

Laj, C., Kissel, C., Mazaud, A., Channell, J.E.T. \& BeER, J. (2000): North Atlantic palaeointensity stack since $75 \mathrm{ka}$ (NAPIS-75) and the duration of the Laschamp Event. - Phil. Trans. Royal Soc. London, 358: 1009-1025; London.

Lang, A., Hatté, C., Rousseau, D.-D., Antoine, P., Fontugne, M., Zöller, L. \& Hambach, U. (2003): High-resolution chronologies for loess: comparing AMS ${ }^{14} \mathrm{C}$ and optical dating results. - Quaternary Science Reviews, 22: 953-959; Oxford. 
Lian, O.B. \& Roberts, R.G. (2006): Dating the Quaternary: progress in luminescence dating of sediments. - Quaternary Science Reviews, 25: 2449-2468; Oxford.

Maher, B.A. \& Thompson, R. (1995): Palaeorainfall reconstruction from pedogenic magnetic susceptibility variations in Chinese loess and paleosols. - Quaternary Research, 44: 383-391; Washington.

Maher, B.A., Thompson, R., \& Zhou, L.P. (1994): Spatial and temporal reconstructions of changes in the Asian palaeomonsoon: a new mineral magnetic approach. - Earth and Planetary Science Letters, 125: 461-471; Amsterdam.

Meng, X., Derbyshire, E. \& Kemp, R.A. (1997): Origin of the magnetic susceptibility signal in loess. - Quaternary Science Reviews, 16: 833839; Oxford.

Moine, O., Rousseau, D.-D., Antoine, P. \& Hatté, C. (2002): Mise en évidence d'événements climatiques rapides par les faunes de mollusques terrestres des loess Weichseliens de Nussloch (Allemagne). - Quaternaire, 13: 209-217; Paris.

Moine, O., Rousseau, D.-D., \& Antoine, P. (2005): Terrestrial molluscan records of Weichselian Lower to Middle Pleniglacial climatic changes from Nussloch loess series (Rhine Valley, Germany). The impact of local factors. - Boreas, $\mathbf{3 4}$ : 363-380; Oslo.

Novothny, Á., Horváth, E. \& Frechen, M. (2002): The loess profile at Albertirsa, Hungary - improvements in loess stratigraphy by luminescence dating. - Quaternary International, 95/96: 155163; Oxford.

Prescott, J.R. \& Hutton, J.T. (1994): Cosmic ray contributions to dose rates for luminescence and ESR dating: large depths and long-term time variations. - Radiation Measurements, 23: 497500; Amsterdam.

RäHLE, W. (2004): Mollusken aus zwei bedeutenden mittel- und jungpleistozänen Lößprofilen des mittleren Neckarraumes (Baden-Württemberg) und ihre ökologisch-stratigraphische Aussage. - In: Kösel, M. (Hrsg): Festschrift zum 60. Geburtstag von E. BiBus. - Tübinger geowiss. Arb., Serie D, 10: 219-240; Tübingen.

Remy, H. (1969) Würmzeitliche Molluskenfaunen aus Lößserien des Rheingaues und des nördlichen Rheinhessens. - Notizbl. hess. L.-Amt Bodenforsch., 97: 98-116; Wiesbaden.

Rousseau, D.-D. (1989): Réponses des malacofaunes terrestres quaternaires aux contraintes climatiques en Europe septentrionale. - Palaeo- geography, Palaeoclimatology, Palaeoecology, 69: 113-124; Amsterdam.

Rousseau, D.-D., Zöller, L. \& Valet, J.-P. (1998): Late Pleistocene Climate Variations at Achenheim, France, Based on a Magnetic Susceptibility and TL Chronology of Loess. - Quaternary Research, 49: 255-263; Washington.

Rousseau, D.-D., Antoine, P., Hatté, C., Lang, A., Zöller, L., Fontugne, M., Orthman, D., Luck, J.M., Moine, O., Labonne, M., Bentaleb, I. \& Jolly, D. (2002): Abrupt millennial climatic change from Nussloch (Germany) Upper Weichselian eolian records during the last Glaciation. - Quaternary Science Reviews, 21: 1577-1582; Oxford.

SABel, K. J. \& Thews, J.-D. (1986): Ein WürmlößProfil in Wiesbaden-Schierstein. - Geol. Jb. Hessen, 114: 111-114; Wiesbaden

Schirmer, W. (1990): Schwalbenberg südlich Remagen. - In: Schirmer, W. (Hrsg.): Rheingeschichte zwischen Mosel und Maas. - deuqua-Führer 1: 105-108; Hannover (DEUQUA).

Schirmer, W. (2000): Eine Klimakurve des Oberpleistozäns aus dem rheinischen Löß. - Eiszeitalter u. Gegenwart, 50: 25-49; Hannover.

Schönhals, E., Rohdenburg, H. \& Semmel, A. (1964): Ergebnisse neuerer Untersuchungen zur Würmlöß-Gliederung in Hessen. - Eiszeitalter u. Gegenwart, 15: 199-206: Öhringen.

Semmel, A. (1967): Neue Fundstellen von vulkanischem Material in hessischen Lössen. - Notizbl. hess. L.-Amt Bodenforsch., 95: 104-108; Wiesbaden.

Semmel, A. (1968): Studien über den Verlauf der jungpleistozäner Formung in Hessen. - Frankfurter geogr. H., 45: 133 S.; Frankfurt a. M.

Semmel, A. (1969): Bemerkungen zur Würmlößgliederung im Rhein-Main-Gebiet. - Notizbl. hess. L-Amt Bodenforsch., 97: 395-399; Wiesbaden.

Semmel, A. (1996): Stop 1, Paläoböden im Würmlöß, insbesondere im Altwürmlöß des Steinbruchs Mainz-Weisenau - Problemstellung und Übersicht über die Forschungsergebnisse. - Frankfurter geowiss. Arb., Serie D, 20: 11-20; Frankfurt a.M.

Semmel, A. (1999): Die paläopedologische Gliederung des älteren Würmlösses in Mitteleuropa - erörtert an Beispielen aus dem Rhein-Maingebiet. - Z. geol. Wiss., 27 (1/2): 221-233; Berlin.

Sommé, J., Lautridou, J.-P., Heim, J., Maucorps, J., Puisségur, J.-J., Rousseau, D.-D., Thévenin, A. \& van Vliet-Lanoe, B. (1986): Le cycle climatique du Pléistocène supérieur dans les loess 
d'Alsace à Achenheim. - Bull. de l'Assoc. franç. pour l'étude du Quaternaire, 1/2: 97-104; Paris.

Terhorst, B., Appel, E. \& Werner, A. (2001): Palaeopedology and magnetic susceptibility of a loess-palaeosol sequence in southwest Germany. - Quaternary International, 76/77: 231-240; Oxford.

WintLe, A.G. (1973): Anomalous fading of thermoluminescence in mineral samples. - Nature, 245: 143-144; London.

WinTLE, A.G. (1997): Luminescence dating: laboratory procedures and protocols. - Radiation Measurements, 27: 769-817; Amsterdam, Oxford.
ZöLLER, L. (1995): Würm- und Rißlöß-Stratigraphie und Thermolumineszenz-Datierung in Süddeutschland und angrenzenden Gebieten. - 208 S.; Heidelberg (unveröff. Habil.-Schr. Univ. Heidelberg).

Zöller, L. \& LösChER, M. (1999): The last glacialinterglacial cycle in the loess section at Nussloch and underlying upper Tertiary loams. - In: WEIDENFELlER, M. \& ZÖlLER, L. (Hrsg.): Loess in the Middle and Upper Rhine area, Field guide Loessfest '99: 37-50; Bonn. 